TI 2012-101/II

Tinbergen Institute Discussion Paper
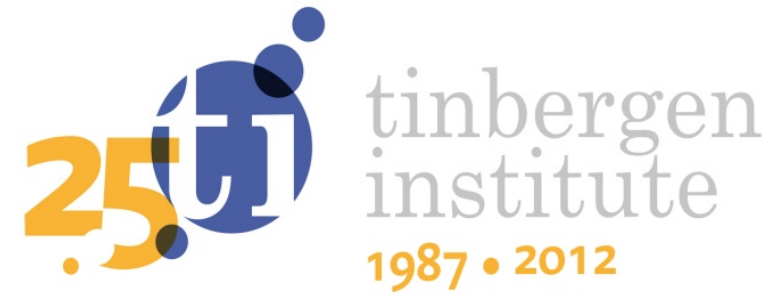

\title{
On the Core of Cost-Revenue Games: Minimum Cost Spanning Tree Games with Revenues
}

\author{
Arantza Estévez-Fernández' \\ Hans Reijnierse ${ }^{2}$
}

${ }^{\prime}$ Faculty of Economics and Business Administration, VU University Amsterdam, and Tinbergen Institute;

2 CentER, Tilburg University. 
Tinbergen Institute is the graduate school and research institute in economics of Erasmus University Rotterdam, the University of Amsterdam and VU University Amsterdam.

More TI discussion papers can be downloaded at http://www.tinbergen.nl

Tinbergen Institute has two locations:

Tinbergen Institute Amsterdam

Gustav Mahlerplein 117

1082 MS Amsterdam

The Netherlands

Tel.: +31(0)205251600

Tinbergen Institute Rotterdam

Burg. Oudlaan 50

3062 PA Rotterdam

The Netherlands

Tel.: +31(0)10 4088900

Fax: $+31(0) 104089031$

Duisenberg school of finance is a collaboration of the Dutch financial sector and universities, with the ambition to support innovative research and offer top quality academic education in core areas of finance.

DSF research papers can be downloaded at: http://www.dsf.nl/

Duisenberg school of finance

Gustav Mahlerplein 117

1082 MS Amsterdam

The Netherlands

Tel.: +31(0)20 5258579 


\title{
On the Core of Cost-Revenue Games: Minimum Cost Spanning Tree Games with Revenues
}

\author{
Arantza Estévez-Fernández ${ }^{\mathrm{a}} \quad$ Hans Reijnierse $^{\mathrm{b}}$
}

\begin{abstract}
In this paper, we analyze cost sharing problems arising from a general service by explicitly taking into account the generated revenues. To this cost-revenue sharing problem, we associate a cooperative game with transferable utility, called cost-revenue game. By considering cooperation among the agents using the general service, the value of a coalition is defined as the maximum net profit that the coalition may obtain by means of cooperation. As a result, a coalition may profit from not allowing all its members to get the service that generates the revenues. We focus on the study of the core of cost-revenue games. Under the assumption that cooperation among the members of the grand coalition grants the use of the service under consideration to all its members, it is shown that a cost-revenue game has a non-empty core for any vector of revenues if, and only if, the dual game of the cost game has a large core. Using this result, we investigate minimum cost spanning tree games with revenues. We show that if every connection cost can take only two values (low or high cost), then, the corresponding minimum cost spanning tree game with revenues has a non-empty core. Furthermore, we provide an example of a minimum cost spanning tree game with revenues with an empty core where every connection cost can take only one of three values (low, medium, or high cost).
\end{abstract}

Keywords: Cost-revenue allocation problem, cooperative game, core, minimum cost spanning tree problem.

\section{JEL Classification Number: C71}

a Tinbergen Institute and Department of Econometrics \& OR, VU University Amsterdam, De Boelelaan 1105, 1081 HV Amsterdam, The Netherlands. E-mail address: arantza.estevezfernandez@vu.nl

${ }^{\mathrm{b}}$ CentER and Department of Econometrics \& OR, Tilburg University, P.O. Box 90153, 5000 LE Tilburg, The Netherlands. E-mail address: j.h.reijnierse@tilburguniversity.edu 


\section{Introduction}

In this paper, we study cost sharing problems arising from a general service by explicitly taking into account the revenues generated by this service. In addition, we apply our findings to minimum cost spanning tree (mcst) problems with revenues.

The study of cost sharing problems combined with revenues has been considered in the literature before. Littlechild and Owen (1976) initiated this stream of literature within the framework of airport problems, where they provide an algorithm to compute the nucleolus of airport profit games. Brânzei, Iñarra, Tijs and Zarzuelo (2006) is a recent follow-up to Littlechild and Owen (1976). Suijs, Borm, Hamers, Quant and Koster (2005) analyze the allocation of costs and revenues within a public network communication structure within the context of cooperative games with transferable utility. Meertens and Potters (2006) provide an algorithm to compute the nucleolus of fixed tree games with revenues. EstévezFernández, Borm, Meertens and Reijnierse (2009) focus on the analysis of the core of routing games with revenues.

Here, we consider the situation in which a group of agents may cooperate to reduce the (possibly nonlinear) costs associated with the performance of a service. Each agent has some fixed revenue from the performance of the service. The question is how to share the net revenues obtained from cooperation among the agents. To solve this allocation problem, we use cooperative game theoretic techniques. By considering cooperation among the agents, we define a cost-revenue game where the value of a coalition is defined as the maximum net profit that the coalition can obtain by means of cooperation. Note that with this definition, a coalition may profit from not allowing all its members to get the service that generates the revenues. In order to provide an allocation of the cost-revenue sharing problem at hand, we focus on the core of the cost-revenue game. The core of a game is the set of allocations of the total net revenues obtained by the cooperation of all agents to which no group of agents has an incentive to deviate, that is, the core is the set of stable allocations of the total net revenue. In this paper, we characterize the class of cost-revenue games with a nonempty core under the assumption that cooperation among the members of the grand coalition grants the use of the service under consideration to all its members. It turns out that a cost-revenue game has a nonempty core for any vector of revenues that guarantees full service to all the members of the grand coalition if, and only if, the dual game of the corresponding cost game has a large core.

We apply the general results found for cost-revenue games to the study of mcst allocation problems with revenues. In an mcst allocation problem, a group of customers needs to be connected to a common information source; the customer needs a physical connection to the source (for instance, the cable for TV transmission) and this connection may be done either directly, or indirectly through other costumers. 
It turns out that the cheapest way to connect all customers to the source is through a spanning tree that can be constructed by means of cooperation among the customers. The mcst allocation problem is, then, how to share the connection costs among the agents. Claus and Kleitman (1973) introduced this problem, which has been extensively analyzed in the literature. Granot and Huberman (1981) define mcst games and show that mcst games have a nonempty core. Granot and Huberman (1984) define allocation rules for mcst allocation problems which lead to elements of the core of the corresponding mcst games and show that, for some instances, some of these rules lead to the nucleolus of the associated game. Kuipers, Solymosi and Aarts (2000) provide a method to compute the nucleolus of an mcst game in polynomial time. For a more extensive overview on the literature of mcst games, we refer to Moretti (2008). When the revenues obtained by the customers by being connected to the source are taking into account, we face an mcst allocation problem with revenues. In this paper, we define mcst games with revenues and we provide an example of an mcst game with revenues where it is profitable that all the members of the grand coalition connect to the source and whose core is empty. In this example, the connection costs between two different players can only take one of three values: high, medium, or low cost. We show that if the connection costs can only take one of two costs (high or low cost), then, the associated mcst game with revenues always has a nonempty core, of course, under the assumption that it is profitable for the grand coalition to have all its members connected to the source.

The remainder of the paper is organized as follows. Section 2 provides the basic definitions and terminology of graph theory and cooperative games used in this paper. In Section 3, we analyze costrevenue games. Section 4 analyzes mcst games with revenues. We conclude with some further remarks in Section 5 .

\section{Preliminaries}

\subsection{Graph theory}

A graph $\Gamma$ is a tuple $(V, E)$ in which $V$ is the finite set of nodes and $E$ is the set of edges. A subgraph $\left(V^{\prime}, E^{\prime}\right)$ of $\Gamma$ is a graph with $V^{\prime} \subset V$ and $E^{\prime}=\left\{\{v, w\} \in E: v, w \in V^{\prime}\right\}$; we denote $\Gamma_{\mid V^{\prime}}=\left(V^{\prime}, E^{\prime}\right)$.

A path from node $v$ to $w$, $\operatorname{path}(v, w)$, is a sequence of nodes $v_{0}, v_{1}, \ldots, v_{p}$ with $v_{0}=v, v_{p}=w$ and, for each $k \in\{0, \ldots, p-1\},\left\{v_{k}, v_{k+1}\right\} \in E$. We denote by $V(\operatorname{path}(v, w))$ the set of nodes of $\operatorname{path}(v, w)$ and by $E(\operatorname{path}(v, w))$ the set of edges of $\operatorname{path}(v, w)$. Formally, $V(\operatorname{path}(v, w))=\left\{v_{0}, v_{1}, \ldots, v_{p}\right\}$ and $E(\operatorname{path}(v, w))=\left\{\left\{v_{0}, v_{1}\right\}, \ldots,\left\{v_{p-1}, v_{p}\right\}\right\}$. A path $v_{0}, \ldots, v_{p}$ is simple if all nodes are different. A cycle, $\kappa$, is a sequence of nodes $v_{0}, v_{1}, \ldots, v_{p}$ with $v_{0}=v_{p}, p \geq 3, v_{0}, v_{1}, \ldots, v_{p-1}$ different nodes and, for each $l \in\{0, \ldots, p-1\},\left\{v_{l}, v_{l+1}\right\} \in E$. We denote by $V(\kappa)$ the set of nodes of $\kappa$ and by $E(\kappa)$ the set of edges of 
$\kappa$. Formally, $V(\kappa)=\left\{v_{0}, v_{1}, \ldots, v_{p-1}\right\}$ and $E(\kappa)=\left\{\left\{v_{0}, v_{1}\right\}, \ldots,\left\{v_{p-1}, v_{p}\right\}\right\}$. Given a graph $\Gamma$, we denote by $\mathbf{C}(\Gamma)$ the set of cycles of $\Gamma$.

A subset $V^{\prime}$ of $V$ is called connected if, for every $v, w \in V^{\prime}$, there is a path from $v$ to $w$ using only nodes of $V^{\prime}$; we denote by $\mathrm{C}(\Gamma)$ the set of all connected subsets of $V$, that is, $\mathcal{C}(\Gamma)=\left\{V^{\prime} \subset V: V^{\prime}\right.$ is connected $\}$. A subset $U$ of $V$ is called maximally connected or component if $U$ is connected and, for any $w \in V \backslash U$, $U \cup\{w\}$ is not connected. Given $V^{\prime} \subset V$, we denote by $V_{\mid \Gamma}^{\prime}$ the set of components of $V^{\prime}$ with respect to $\Gamma$.

A graph $\Gamma=(V, E)$ is a forest if it has no cycles and it is a tree if it is a connected forest. A rooted tree with root $v_{0} \in V$ is a tree where node $v_{0}$ is singled out.

\subsection{Cooperative games}

A cooperative (transferable utility) game in characteristic function form is an ordered pair $(N, v)$ where $N$ is a finite set of players and $v: 2^{N} \rightarrow \mathbb{R}$ is the characteristic function satisfying $v(\emptyset)=0$. In general, $v(S)$ represents the value of coalition $S$, that is, the joint payoff that this coalition can obtain when its members decide to cooperate. A cooperative game can reflect costs or rewards. A game reflecting costs is denoted by a mapping $c$, while a game reflecting rewards is denoted by a mapping $v$. The following properties and definitions refer to rewards.

The central question within a cooperative framework is how to share the revenues that the players obtain by means of cooperation, that is, how to share the value of the grand coalition, $v(N)$, among the players. One of the most studied solution concepts in cooperative game theory is the core of a game, first introduced in Gillies (1953). The core of a game $(N, v)$, Core $(v)$, is the set of efficient allocations of $v(N)$ (or in other words, exactly $v(N)$ is shared among the players) that are coalitionally rational (that is, to which no coalition can reasonably object). Formally, ${ }^{1}$

$$
\operatorname{Core}(v)=\left\{x \in \mathbb{R}^{N}: x(N)=v(N), x(S) \geq v(S) \text { for all } S \subset N\right\} .
$$

The upper core of a game $(N, v), \mathrm{U}(v)$, is the set of not necessarily efficient allocations that are coalitionally rational. Formally,

$$
\mathrm{U}(v)=\left\{x \in \mathbb{R}^{N}: x(S) \geq v(S) \text { for all } S \subset N\right\}
$$

Largeness of the core was first introduced in Sharkey (1982). The core of $(N, v)$ is said to be large if, for every $x \in \mathrm{U}(v)$, there is a $y \in \operatorname{Core}(v)$ with $y_{i} \leq x_{i}$ for each $i \in N$. The class of games with a large core has been characterized in Estévez-Fernández (2012) by means of minimal cover inequalities. Given a finite set $N$, a collection $\mathcal{C} \subset 2^{N} \backslash\{\emptyset\}$ is called a minimal cover of $N$ if $\mathcal{C} \neq\{N\}$ and the following two conditions are satisfied:

\footnotetext{
${ }^{1}$ Here and further, for any vector $x \in \mathbb{R}^{N}$, we denote $x(S):=\sum_{i \in S} x_{i}$.
} 
(i) $\cup_{S \in \mathcal{C}} S=N$;

(ii) $\cup_{S \in \mathcal{C} \backslash\left\{S^{\prime}\right\}} S \neq N$ for every $S^{\prime} \in \mathcal{C}$.

By condition (i), it follows that $\mathcal{C}$ covers $N$; by condition (ii), we have that no element of $\mathcal{C}$ is superfluous. We denote by $\mathbf{M C}(N)$ the set of minimal covers of $N$. Let $\mathcal{P}(N)$ denote the set of partitions of $N$. Note that $\mathcal{P}(N) \backslash\{\{N\}\} \subset \mathbf{M C}(N)$.

Given a finite set $N$ and $\mathcal{C} \in \mathbf{M C}(N)$, for every $i \in N$, we denote by $\xi(\mathcal{C}, i)$ the number of times that $i$ is "extra covered" in $\mathcal{C}$; formally, $\xi(\mathcal{C}, i)=|\{S \in \mathcal{C}: i \in S\}|-1$. We denote by $E(\mathcal{C})$ the set of elements of $N$ that are "extra covered" by $\mathcal{C}$; formally, $E(\mathcal{C})=\{i \in N: \xi(\mathcal{C}, i)>0\}$.

Given $\mathcal{C} \in \mathbf{M C}(N)$ with $E(\mathcal{C}) \neq \emptyset$, a vector $\mu \in \mathbb{R}_{+}^{2^{E(\mathcal{C})} \backslash\{\emptyset\}}$ is said to be a vector of minimal cover balanced weights (mc-balanced vector) for $\mathcal{C}$ if

$$
\sum_{S \in 2^{E(\mathcal{C})} \backslash\{\emptyset\}} \mu_{S} e^{S}=\sum_{i \in E(\mathcal{C})} \xi(\mathcal{C}, i) e^{\{i\}} .
$$

with $e^{R} \in \mathbb{R}^{N}$ defined as $e_{i}^{R}=1$ if $i \in R$ and $e_{i}^{R}=0$ if $i \notin R$ for every $R \in 2^{\mathrm{N}} \backslash\{\emptyset\}$. We denote by $M(\mathcal{C})$ the set of mc-balanced vectors for $\mathcal{C}$. Trivially, $M(\mathcal{C}) \neq \emptyset$ if $E(\mathcal{C}) \neq \emptyset$. It follows that

$$
\sum_{S \in \mathcal{C}} e^{S}=e^{N}+\sum_{S \in 2^{E(\mathcal{C})} \backslash\{\emptyset\}} \mu_{S} e^{S}
$$

for every $\mu \in M(\mathcal{C})$. Note that if $E(\mathcal{C})=\emptyset$, then, $\mathcal{C}$ is a non-trivial partition of $N$ and $\sum_{S \in \mathcal{C}} e^{S}=e^{N}$. Given a game $(N, v)$ and a minimal cover $\mathcal{C} \in \mathbf{M C}(N)$, the minimal cover inequality associated with $\mathcal{C}$ is

$$
\sum_{S \in \mathcal{C}} v(S) \leq v(N)+\max _{\mu \in M(\mathcal{C})}\left\{\sum_{S \in 2^{E(\mathcal{C})} \backslash\{\emptyset\}} \mu_{S} v(S)\right\}
$$

Theorem 2.1 (Estévez-Fernández (2012)). A game $(N, v)$ has a large core if, and only if, all minimal cover inequalities are satisfied.

Let $(N, v)$ be a game, a coalition $S \subset N$ is called essential if, for every non-trivial partition $\left\langle S_{1}, \ldots, S_{t}\right\rangle$ of $S, v(S)>\sum_{l=1}^{t} v\left(S_{l}\right)$. We denote by $\operatorname{Ess}(v)$ the set of essential coalitions of $(N, v)$. Notice that only essential coalitions are needed for the description of both the core and the upper core of a game. Therefore, following the comments in the Final Remarks of Estévez-Fernández (2012), we can rewrite Theorem 2.1 as follows:

A game $(N, v)$ has a large core if, and only if,

$$
\sum_{S \in \mathcal{C}} v(S) \leq v(N)+\max _{\mu \in M(\mathcal{C})}\left\{\sum_{S \in 2^{E(\mathcal{C})} \backslash\{\emptyset\}} \mu_{S} v(S)\right\}
$$

for every $\mathcal{C} \in \mathbf{M C}(N)$ such that $\mathcal{C} \subset \operatorname{Ess}(v)$. 
A game $(N, v)$ is said to be exact (Schmeidler 1972) if, for every $S \subset N$, there exists $x \in \operatorname{Core}(v)$ such that $x(S)=v(S)$.

Given a forest $\Gamma=(N, E)$, the game $(N, v)$ is said to be forest-essential with respect $t^{2} \Gamma$ if $v(N)=\max _{\left\langle S_{1}, \ldots, S_{t}\right\rangle \in \mathcal{P}(N)}\left\{\sum_{l=1}^{t} v\left(S_{l}\right)\right\}$ and all essential coalitions are connected with respect to $\Gamma$, that is, $\operatorname{Ess}(v) \subset \mathcal{C}(\Gamma)$. If $\Gamma$ is a tree, we say that $(N, v)$ is tree-essential with respect to $\Gamma$.

Let $(N, c)$ be a cost game. The anti core of $(N, c)$, ACore $(c)$, is the set of efficient allocations of $c(N)$ to which no coalition can reasonably object. Formally,

$$
\operatorname{ACore}(c)=\left\{x \in \mathbb{R}^{N}: x(N)=c(N), x(S) \leq c(S) \text { for all } S \subset N\right\} .
$$

The dual game of $(N, c),\left(N, c^{*}\right)$, is defined, for every $S \subset N$, by

$$
c^{*}(S)=c(N)-c(N \backslash S) .
$$

It is easily seen that $\operatorname{ACore}(c)=\operatorname{Core}\left(c^{*}\right)$.

\section{Cost-revenue games}

In this section, we analyze situations in which a group of agents cooperate in order to obtain a higher joint revenue. Each time a coalition decides to cooperate, an additive reward is obtained and a (possibly nonadditive) cost is generated. The problem at hand is how to share the net revenue that is obtained by the grand coalition among the agents. We define cost-revenue games and concentrate on the study of their cores. It turns out that a cost-revenue game has a nonempty core for any vector of revenues that provides maximal cooperation if, and only if, the dual game of the cost game has a large core.

Let $(N, c)$ be a cost game. Consider the case in which, next to the costs that a coalition must confront, the coalition also obtains revenues when it decides to cooperate. Let $b \in \mathbb{R}_{+}^{N}$ be the vector of revenues, where $b_{i}$ is the revenue that player $i$ generates if $i$ gets the service under consideration. Then, the total revenue that a coalition $S \subset N$ can obtain by cooperation is

$$
\pi_{b}(S)=b(S)-c(S)
$$

Notice that, due to the revenue structure of the game, it may be more profitable for coalition $S$ not to form as a whole. Given a cost game $(N, c)$ and a vector of revenues $b \in \mathbb{R}_{+}^{N}$, we define the cost-revenue game, $\left(N, v_{b}\right)$, for every $S \subset N$, by

$$
v_{b}(S)=\max _{R \subset S}\left\{\pi_{b}(R)\right\} .
$$

\footnotetext{
${ }^{2}$ In fact, a forest-essential game with respect to $\Gamma$ is a game with the total dependency property (see Kuipers, Solymosi and Aarts (2000)) and where the grand coalition is stable (see Derks and Kuipers (1997)).
} 
The following example illustrates the computation of cost-revenues games and shows that full participation of the grand coalition is relevant in order to have nonemptiness of the core of the corresponding cost-revenue game.

Example 3.1. Consider the six players cost game $(N, c)$ with characteristic function defined as follows:

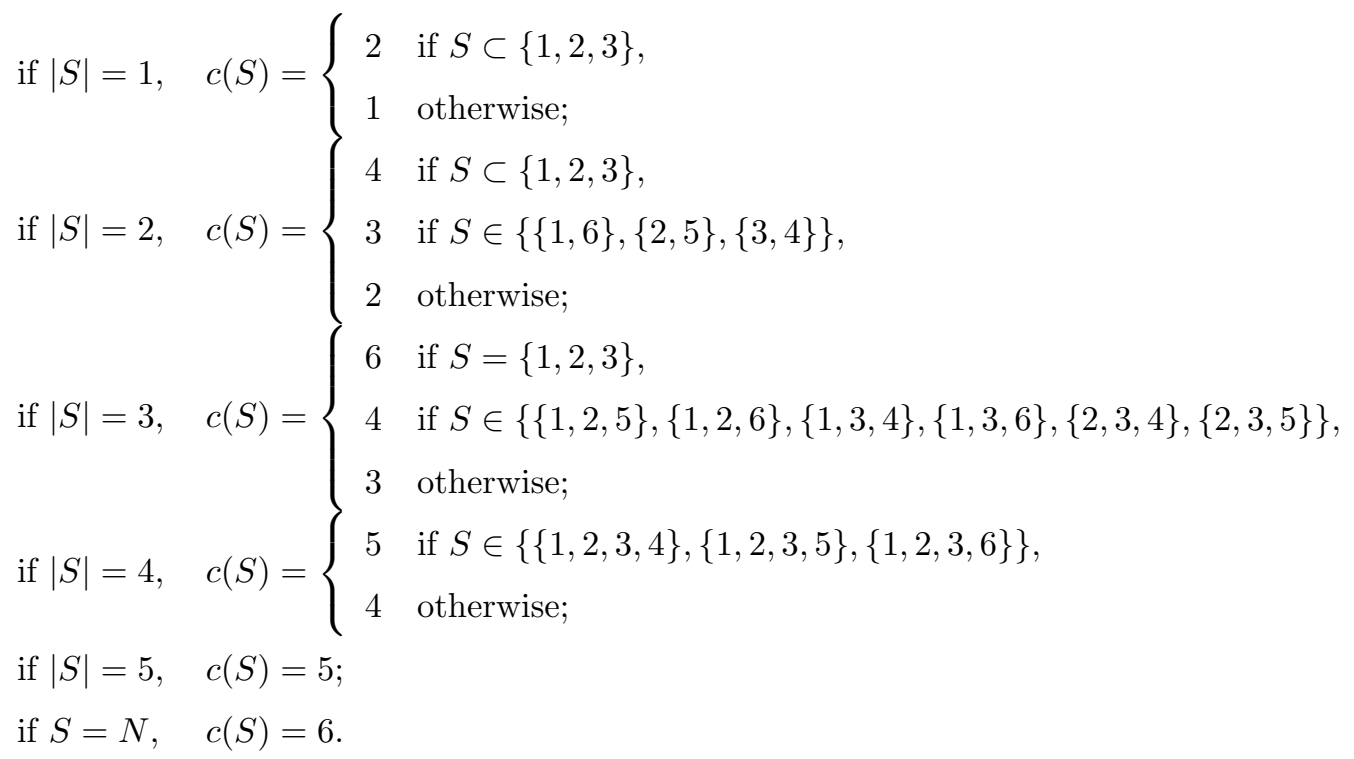

Let $b=(2,2,2,0,0,0)$. The cost-revenue game is, then,

$$
v_{b}(S)= \begin{cases}1 & \text { if }\{1,2,4\} \subset S, \text { or }\{1,3,5\} \subset S, \text { or }\{2,3,6\} \subset S, \\ 0 & \text { otherwise }\end{cases}
$$

Note that $v_{b}(N)=1>0=b(N)-c(N)$. Below, we explain how to compute the value of coalition $\{1,2,4\}$.

$$
\begin{aligned}
v_{b}(\{1,2,4\}) & =\max \left\{\pi_{b}(\{\emptyset\}), \pi_{b}(\{1\}), \pi_{b}(\{2\}), \pi_{b}(\{4\}), \pi_{b}(\{1,2\}), \pi_{b}(\{1,4\}), \pi_{b}(\{2,4\}), \pi_{b}(\{1,2,4\})\right\} \\
& =\max \{0,2-2,2-2,0-1,4-4,2-2,2-2,4-3\}=\max \{0,0,0,-1,0,0,0,1\}=1 .
\end{aligned}
$$

Note that $(N, c)$ has a nonempty anti core since, for instance, $(1,1,1,1,1,1) \in \operatorname{ACore}(c)$. However, $\left(N, v_{b}\right)$ has an empty core. To show this, suppose that $x \in \operatorname{Core}\left(v_{b}\right)$, then, $1 \leq x_{1}+x_{2}+x_{4}, 1 \leq x_{1}+x_{3}+x_{5}$, $1 \leq x_{2}+x_{3}+x_{6}, 0 \leq x_{4}+x_{5}+x_{6}$. By adding all these equations, we obtain $3 \leq 2 x(N)=2 v_{b}(N)=2$, establishing a contradiction; then, $\operatorname{Core}\left(v_{b}\right)=\emptyset$. Here, the first equality follows from $x \in \operatorname{Core}\left(v_{b}\right)$.

As we have seen in the example above, when no all members of the grand coalition get the service that generates the revenues, the core of the cost-revenue game can be empty. For that reason, we assume 
from now on that it is profitable to form the grand coalition, that is,

$$
v_{b}(N)=\pi_{b}(N)=b(N)-c(N)
$$

Taking into account that $\pi_{b}(N)=\max _{R \subset N}\{b(R)-c(R)\}$, it follows that Assumption (3.1) is equivalent to

$$
b(S) \geq c^{*}(S) \text { for every } S \subset N
$$

Assumption (3.1) may seem somehow restrictive. In fact, Equation (3.1) ensures that the grand coalition will effectively form without leaving any player behind. The following example shows that nonemptyness of the anti core of the cost game is not sufficient to have nonemptyness of the core of the corresponding cost-revenue game, even when the cost game is exact. ${ }^{3}$ Therefore, revenues have a real impact in the structure of the core of cost-revenue games.

Example 3.2. Consider the five players exact cost game $(N, c)$ with ${ }^{4} \operatorname{ACore}(c)=\operatorname{conv}\{(0,0,1,2,0)$, $(1,1,0,0,1)\}$, and let $b=(1,1,1,1,0)$. In Table 1 , we provide the coalitional values of $(N, c),\left(N, c^{*}\right)$,

\begin{tabular}{|c|c|c|c|c|c|c|c|c|c|c|c|c|c|c|c|}
\hline$S$ & $\{1\}$ & $\{2\}$ & $\{3\}$ & $\{4\}$ & $\{5\}$ & $\{1,2\}$ & $\{1,3\}$ & $\{1,4\}$ & $\{1,5\}$ & $\{2,3\}$ & $\{2,4\}$ & $\{2,5\}$ & $\{3,4\}$ & $\{3,5\}$ & $\{4,5\}$ \\
\hline$c(S)$ & 1 & 1 & 1 & 2 & 1 & 2 & 1 & 2 & 2 & 1 & 2 & 2 & 3 & 1 & 2 \\
\hline$c^{*}(S)$ & 0 & 0 & 0 & 0 & 0 & 0 & 1 & 1 & 0 & 1 & 1 & 0 & 0 & 1 & 1 \\
\hline$b(S)$ & 1 & 1 & 1 & 1 & 0 & 2 & 2 & 2 & 1 & 2 & 2 & 1 & 2 & 1 & 1 \\
\hline$v_{b}(S)$ & 0 & 0 & 0 & 0 & 0 & 0 & 1 & 0 & 0 & 1 & 0 & 0 & 0 & 0 & 0 \\
\hline$S$ & \multicolumn{2}{|c|}{$1,2,3$} & $\{1,2,4\}$ & \multicolumn{2}{|c|}{$1,2,5$} & $\{1,3,4\}$ & \multicolumn{2}{|c|}{$1,3,5$} & $\{1,4,5\}$ & $\{2,3,4\}$ & $\{2,3,5\}$ & \multicolumn{2}{|c|}{$2,4,5$} & $\{3,4,5\}$ & \\
\hline$c(S)$ & \multicolumn{2}{|c|}{2} & 2 & \multicolumn{2}{|c|}{3} & 3 & \multicolumn{2}{|c|}{2} & 2 & 3 & 2 & \multicolumn{2}{|c|}{2} & 3 & \\
\hline$c^{*}(S)$ & \multicolumn{2}{|c|}{1} & \multicolumn{2}{|l|}{2} & 0 & \multicolumn{2}{|l|}{1} & 1 & 2 & 1 & 1 & \multicolumn{2}{|c|}{2} & 1 & \\
\hline$b(S)$ & \multicolumn{2}{|c|}{3} & \multicolumn{2}{|l|}{3} & 2 & \multicolumn{2}{|l|}{3} & 2 & 2 & 3 & 2 & & 2 & 2 & \\
\hline$v_{b}(S)$ & 1 & & 1 & & 0 & 1 & & 1 & 0 & 1 & 1 & & 0 & 0 & \\
\hline$S$ & $\{1,2$ & $3,4\}$ & $\{1,2$, & $3,5\}$ & $\{1,2$ & $, 4,5\}$ & $\{1,3,4$, & & $\{2,3,4,5\}$ & $N$ & & & & & \\
\hline$c(S)$ & : & 3 & 3 & 3 & & 3 & 3 & & 3 & 3 & & & & & \\
\hline$c^{*}(S)$ & & 2 & 1 & 1 & & 2 & 2 & & 2 & 3 & & & & & \\
\hline$b(S)$ & & 4 & 3 & 3 & & 3 & 3 & & 3 & 4 & & & & & \\
\hline$v_{b}(S)$ & & L & 1 & 1 & & 1 & 1 & & 1 & 1 & & & & & \\
\hline
\end{tabular}
$(N, b)$ and $\left(N, v_{b}\right)$.

Table 1: Coalitional values of the cost game, dual game, additive revenues, and cost-revenue game in Example 3.2.

Note that $b(S) \geq c^{*}(S)$ for every $S \subset N$. However, $\left(N, v_{b}\right)$ has an empty core. To show this, suppose that $x \in \operatorname{Core}\left(v_{b}\right)$, then, $1 \leq x_{1}+x_{2}+x_{3}+x_{4}, 1 \leq x_{1}+x_{2}+x_{3}+x_{5}, 1 \leq x_{1}+x_{2}+x_{4}+x_{5}, 1 \leq x_{1}+x_{3}+x_{4}+x_{5}$,

\footnotetext{
${ }^{3}$ Similarly as in (reward) games, a cost game is exact if, for every $S \subset N$, there is an $x \in$ ACore $(c)$ such that $x(S)=c(S)$.

${ }^{4} \mathrm{By}$ conv we refer to the convex hull of the corresponding vectors.
} 
$1 \leq x_{2}+x_{3}+x_{4}+x_{5}$. By adding all these equations, we obtain $5 \leq 4 x(N)=4 v_{b}(N)=4$, establishing a contradiction; then, $\operatorname{Core}\left(v_{b}\right)=\emptyset$. Here, the first equality follows from $x \in \operatorname{Core}\left(v_{b}\right)$.

The first result of this section states that if the core of a cost-revenue game is nonempty, then, its core can be expressed in terms of the vector of revenues and the anti core of the cost game.

Lemma 3.1. Let $(N, c)$ be a cost game, let $b \in \mathbb{R}_{+}^{N}$ be a vector of revenues satisfying Assumption (3.1), and let $\left(N, v_{b}\right)$ be the corresponding cost-revenue game. Then,

$$
\operatorname{Core}\left(v_{b}\right)=\{b-y: y \in \operatorname{ACore}(c) \text { with } y \leq b\}
$$

Proof: We first show " $\subset$ ". If $\operatorname{Core}\left(v_{b}\right)=\emptyset$, we are done. Let $x \in \operatorname{Core}\left(v_{b}\right)$ and let $y=b-x$, we have to show that (i) $y \in \operatorname{ACore}(c)$ and (ii) $y \leq b$.

(i) Let $S \subset N$, then,

$$
b(S)-c(S) \leq \max _{R \subset S}\{b(R)-c(R)\}=v_{b}(S) \leq x(S),
$$

where the last inequality follows because $x \in \operatorname{Core}\left(v_{b}\right)$. Then,

$$
y(S)=b(S)-x(S) \leq c(S)
$$

Besides,

$$
b(N)-c(N)=v_{b}(N)=x(N)
$$

where the first equality follows by Assumption (3.1) and the second one because $x \in \operatorname{Core}\left(v_{b}\right)$. Then,

$$
y(N)=b(N)-x(N)=c(N)
$$

By Equations (3.3) and (3.4), we have that $y \in \operatorname{ACore}(c)$.

(ii) Since $x \in \operatorname{Core}\left(v_{b}\right)$, it follows that, for every $i \in N$,

$$
x_{i} \geq v_{b}(\{i\}) \geq 0
$$

and, therefore, $y_{i}=b_{i}-x_{i} \leq b_{i}$ for every $i \in N$.

Next, we show " $\supset$ ". If there exists no $y \in \operatorname{ACore}(c)$ satisfying $y \leq b$, then, we are done. Let $x=$ $b-y$ with $y \in \operatorname{ACore}(c)$ and $y \leq b$, we have that

$$
x(N)=b(N)-y(N)=b(N)-c(N)=v_{b}(N),
$$


where the second equality follows because $y \in \operatorname{ACore}(c)$ and the third one is a direct consequence of Assumption (3.1). Moreover, for every $S \subset N$, let $R^{S} \subset S$ be such that $v_{b}(S)=\pi_{b}\left(R^{S}\right)=b\left(R^{S}\right)-c\left(R^{S}\right)$. We have that

$$
x(S) \geq x\left(R^{S}\right)=b\left(R^{S}\right)-y\left(R^{S}\right) \geq b\left(R^{S}\right)-c\left(R^{S}\right)=v_{b}(S),
$$

where the first inequality is a direct consequence of $x \geq 0$ since $b \geq y$ and the fact that $R^{S} \subset S$, and the second inequality follows since $y \in \operatorname{ACore}(c)$. By Equations (3.5) and (3.6), we have that $x \in \operatorname{Core}\left(v_{b}\right)$.

The following result characterizes the set of cost games in which, for any vector of revenues satisfying Assumption (3.1), it follows that the corresponding cost-revenue game has a nonempty core.

Theorem 3.2. Let $(N, c)$ be a cost game. Then, $\left(N, v_{b}\right)$ has a nonempty core for any $b \in \mathbb{R}_{+}^{N}$ satisfying Assumption (3.1) if, and only if, $\left(N, c^{*}\right)$ has a large core.

Before starting the proof, notice that $b \in \mathbb{R}_{+}^{N}$ satisfies Assumption (3.1) (or, equivalently, Equation (3.2): $b(S) \geq c^{*}(S)$ for every $\left.S \subset N\right)$ if, and only if, $b \in \mathrm{U}\left(c^{*}\right)$.

Proof: We first show the "if" part. Let $\left(N, c^{*}\right)$ have a large core. Then, for any $b \in \mathrm{U}\left(c^{*}\right)$, there is a $y^{b} \in \operatorname{Core}\left(c^{*}\right)=\operatorname{ACore}(c)$ with $b \geq y^{b}$. By Lemma 3.1, it follows that $b-y^{b} \in \operatorname{Core}\left(v_{b}\right)$.

We now show the "only if" part. Let $(N, c)$ be such that $\left(N, v_{b}\right)$ has a nonempty core for every $b \in \mathbb{R}_{+}^{N}$ satisfying Assumption (3.1). Let $b \in \mathrm{U}\left(c^{*}\right)$ and choose any $x \in \operatorname{Core}\left(v_{b}\right)$. First, we have that $b \geq b-x$ since $x \geq 0$ by non-negativity of $\left(N, v_{b}\right)$; second, $b-x \in \operatorname{ACore}(c)=\operatorname{Core}\left(c^{*}\right)$ by Lemma 3.1. Therefore, $\left(N, c^{*}\right)$ has a large core.

The next example shows that largeness of the core of $\left(N, c^{*}\right)$ and largeness of the anti core ${ }^{5}$ of $(N, c)$ can be unrelated.

Example 3.3. Consider the cost game $(N, c)$ given in Table 2.

\begin{tabular}{|l|c|c|c|c|c|c|c|c|c|c|c|c|c|c|c|}
\hline$S$ & $\{1\}$ & $\{2\}$ & $\{3\}$ & $\{4\}$ & $\{1,2\}$ & $\{1,3\}$ & $\{1,4\}$ & $\{2,3\}$ & $\{2,4\}$ & $\{3,4\}$ & $\{1,2,3\}$ & $\{1,2,4\}$ & $\{1,3,4\}$ & $\{2,3,4\}$ & $N$ \\
\hline$c(S)$ & 2 & 2 & 1 & 1 & 4 & 2 & 2 & 2 & 2 & 2 & 3 & 3 & 3 & 3 & 4 \\
\hline$c^{*}(S)$ & 1 & 1 & 1 & 1 & 2 & 2 & 2 & 2 & 2 & 0 & 3 & 3 & 2 & 2 & 4 \\
\hline
\end{tabular}

Table 2: Coalitional values of the cost game and dual game in Example 3.3.

\footnotetext{
${ }^{5}$ We define the lower core of a cost game $(N, c), \mathrm{L}(c)$, as the set of non necessarily efficient allocations to which no coalition can reasonably object. Formally, $\mathrm{L}(c)=\left\{x \in \mathbb{R}^{N}: x(S) \leq c(S)\right.$ for all $\left.S \subset N\right\}$. Then, we say that the cost game $(N, c)$ has a large anti core if, for any $x \in \mathrm{L}(c)$, there is a $y \in \operatorname{ACore}(c)$ with $x_{i} \leq y_{i}$ for each $i \in N$.
} 
It can be easily seen that $\operatorname{Core}\left(c^{*}\right)=\operatorname{ACore}(c)=\{(1,1,1,1)\}$. Since, for any $x \in \mathrm{U}\left(c^{*}\right)$, we have that $x_{i} \geq 1$ for every $i \in N$, it follows that the core of $\left(N, c^{*}\right)$ is large. On the other hand, it can be checked that $(1,1.1,0.9,0.9) \in \mathrm{L}(c)$, and, therefore, the anticore of $(N, c)$ is not large since $1.1>1$.

\section{Minimum cost spanning tree games with revenues}

In this section, we study minimum cost spanning tree (mcst) games with revenues. First, we provide an example of an mcst game with revenues that has an empty core. In this example, the connection cost between two different villages can only take one of three possible values (low, medium, and high cost). Therefore, the arising question is whether we can find an mcst game with revenues with an empty core in which the connection cost between two different villages can only take one of, at most, two possible values (low and high cost). We call these games 2-mcst games with revenues. Second, we answer this question by showing that, under Assumption (3.1), every 2-mcst game with revenues has a non-empty core.

Let $N=\{1,2, \ldots, n\}$ denote the set of villages that have to be connected to a source. Let $N_{0}=N \cup\{0\}$, where 0 denotes the source. Let $C=\left(c_{i j}\right)$ be an $N_{0} \times N_{0}$-matrix, where $c_{i j} \geq 0$, for every $i, j \in N_{0}$, represents the costs to go from village $i$ to village $j$. Since the cost matrix is nonnegative, it turns out that the graph that connects all villages to the source with a minimum cost is a tree. Throughout this article we assume that:

$$
\begin{aligned}
& \text { (i) } c_{i i}=0 \text { for all } i \in N_{0}, \\
& \text { (ii) } c_{i j}=c_{j i} \text { for all } i, j \in N_{0} \quad \text { (symmetry). }
\end{aligned}
$$

The network $\left(N_{0}, C\right)$ is usually represented by the complete graph on $N_{0}$ with costs $c_{i j}$ on the edge between nodes $i$ and $j$.

Given a network $\left(N_{0}, C\right)$ and a subset $S \subset N$, we denote $S_{0}:=S \cup\{0\}$. A tree $(V, E)$ is called a spanning tree of $S$ if $V=S_{0}$. Let $\mathrm{T}(S)$ denote the set of all spanning trees of $S$ and, for $\Gamma=\left(S_{0}, E\right) \in \mathrm{T}(S)$, let $c(\Gamma, S)$ denote the cost associated with the tree, that is,

$$
c(\Gamma, S)=\sum_{\{i, j\} \in E} c_{i j} .
$$

By associating each village in $N$ with a player, an mcst game, $(N, c)$, is defined, for every $S \subset N$, by

$$
c(S):=\min _{\Gamma \in \mathrm{T}(S)}\{c(\Gamma, S)\} .
$$

Next, consider that if village $i \in N$ is connected to the source, a revenue $b_{i} \geq 0$ is obtained. We denote an mcst problem with revenues by a tuple $\left(N_{0}, C, b\right)$. Associating each village in $N$ with a player, 
we denote by $\left(N, v_{b}\right)$ the corresponding mcst game with revenues. Note that, under Assumption (3.1), we have that $v_{b}(N)=b(N)-c(N)$.

Example 4.1. Consider the mcst problem with revenues represented in Figure 1, where the numbers at the edges represent the connecting costs and the boldface numbers at the nodes represent the revenues.

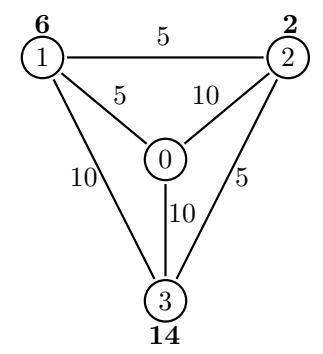

Figure 1: The mcst problem with revenues in Example 4.1.

The value of coalition $\{1,2\}$ is computed as follows:

$$
\begin{aligned}
v_{b}(\{1,2\}) & =\max \left\{0, b_{1}-c(\{1\}), b_{2}-c(\{2\}), b_{1}+b_{2}-c(\{1,2\})\right\} \\
& =\max \{0,6-5,2-10,6+2-10\}=\max \{0,1,-8,-2\}=1
\end{aligned}
$$

The associated mcst game with revenues is given in Table 3.

\begin{tabular}{|l|c|c|c|c|c|c|c|}
\hline$S$ & $\{1\}$ & $\{2\}$ & $\{3\}$ & $\{1,2\}$ & $\{1,3\}$ & $\{2,3\}$ & $N$ \\
\hline$v_{b}(S)$ & 1 & 0 & 4 & 1 & 5 & 4 & 7 \\
\hline
\end{tabular}

Table 3: Coalitional values of the mcst game with revenues in Example 4.1.

It can be checked that $\operatorname{Core}\left(v_{b}\right)=\operatorname{conv}\{(3,0,4),(1,2,4),(1,0,6)\}$.

In general, mcst games with revenues satisfying Assumption (3.1) can have an empty core as the following example illustrates.

Example 4.2. Consider the mcst network $\left(N_{0}, C\right)$ with $N=\{1,2,3,4,5,6,7\}$ and matrix cost defined as

$$
c_{i j}=\left\{\begin{aligned}
0 & \text { if } i=j, \\
3 & \text { if }\{i, j\} \in\{\{0,1\},\{1,2\},\{1,3\},\{1,4\},\{2,7\},\{3,6\},\{4,5\}\}, \\
4 & \text { if }\{i, j\} \in\{\{0,2\},\{0,3\},\{0,4\},\{2,5\},\{3,7\},\{4,6\}\}, \\
5 & \text { otherwise. }
\end{aligned}\right.
$$


Note that $\left(N, c^{*}\right)$ does not have a large core by considering the minimal cover $\mathcal{C}=\{\{1,2,4,5\},\{1,2,3,7\}$, $\{1,3,4,6\}\}$. It follows from $c^{*}(\{1,2,4,5\})=c^{*}(\{1,2,3,7\})=c^{*}(\{1,3,4,6\})=10, c^{*}(N)=21$ and $c^{*}(\{1,2,4,5\})+c^{*}(\{1,2,3,7\})+c^{*}(\{1,3,4,6\})=30>21+8=c^{*}(N)+\max _{\mu \in M(\mathcal{C})}\left\{\sum_{S \in 2^{E(\mathcal{C})} \backslash\{\emptyset\}} \mu_{S} c^{*}(S)\right\}$, where an optimal solution to the maximization problem above is $\hat{\mu}$ defined as $\hat{\mu}_{S}=1$ if $S \in\{\{1,2,3\},\{1,4\}\}$ and $\hat{\mu}_{S}=0$ otherwise. It can be checked that $b=(1,2,2,2,5,5,5)$ satisfies Assumption (3.1) and $\left(N, v_{b}\right)$ has an empty core since $v_{b}(\{4\})=v_{b}(\{1,2\})=v_{b}(\{1,3\})=0, v_{b}(\{2,5,7\})=v_{b}(\{3,6,7\})=$ $v_{b}(\{4,5,6\})=v_{b}(N)=1$ and, therefore, if $x \in \operatorname{Core}\left(v_{b}\right)$, we have that $0 \leq x_{4}, 0 \leq x(\{1,2\}), 0 \leq x(\{1,3\})$, $1 \leq x(\{2,5,7\}), 1 \leq x(\{3,6,7\}), 1 \leq x(\{4,5,6\})$ and $x(N)=1$; then, by adding the inequalities, we obtain $3 \leq 2 x(N)=2$, establishing a contradiction.

Note that every connection cost in the mcst network of Example 4.2 can take one of three given values: 3,4 , or 5 . Next, we analyze the case in which connection costs between two different villages in an mcst network can take, at most, two values: low and high cost. It turns out that, under Assumption (3.1), the corresponding mcst game with revenues always has a non-empty core. From now on, we refer to this type of mcst network as a 2-mcst network. Formally, $\left(N_{0}, C\right)$ is a 2-mcst network if, for every $i, j \in N_{0}, i \neq j$, $c_{i j} \in\{k, K\}$ with $0 \leq k \leq K$.

Next, we show nonemptiness of the core of 2-mcst games with revenues under Assumption (3.1). For this, the dual game of a 2-mcst game plays a crucial role. Therefore, we first provide some results on the dual game of a 2-mcst game. The first result states that the dual game of an mcst game is tree-essential with respect to any optimal tree $\hat{\Gamma}$ for the grand coalition. It is based on results from Kuipers (1994) (see Theorem 4.4 in Kuipers (1994) and comments below) and the proof is, therefore, omitted.

Theorem 4.1. Let $\left(N_{0}, C\right)$ be an mcst network and let $(N, c)$ be the associated mcst game. Let $\hat{\Gamma}$ be an optimal mcst for $N$. Then, the associated dual game $\left(N, c^{*}\right)$ is forest-essential with respect to $\hat{\Gamma}_{\mid N}$.

Before stating the following result, we need to introduce some extra notation. Given a 2-mcst network $\left(N_{0}, C\right)$, we define the $k$-graph $\Gamma^{k}=\left(N_{0}, E^{k}\right)$ as

$$
E^{k}=\left\{\{i, j\} \subset N_{0}: c_{i j}=k\right\}
$$

Note that if $\Gamma^{k}$ is a forest, then, $E^{k}$ is contained in the set of edges of every mcst for $N$. If $\Gamma^{k}$ is not a forest, for every mcst for $N$, there exist some edges $e_{1}, \ldots, e_{t} \in \cup_{\kappa \in \mathbf{C}\left(\Gamma^{k}\right)} E(\kappa)$ such that $E^{k} \backslash\left\{e_{1}, \ldots, e_{t}\right\}$ is contained in the set of edges of this mcst for $N$. Conversely, we can select edges $e_{1}, \ldots, e_{t} \in \cup_{\kappa \in \mathbf{C}\left(\Gamma^{k}\right)} E(\kappa)$ such that $\tilde{\Gamma}=\left(N_{0}, E^{k} \backslash\left\{e_{1}, \ldots, e_{t}\right\}\right)$ is a forest with $\left|N_{0 \mid \tilde{\Gamma}}\right|=\left|N_{0 \mid \Gamma^{k}}\right|$. Then, there exists an mcst for 
$N$ whose set of edges contains $E^{k} \backslash\left\{e_{1}, \ldots, e_{t}\right\}$. Given a 2-mcst network $\left(N_{0}, C\right)$, we define the graph $\Phi^{k}=\left(N_{0}, F^{k}\right)$ as

$$
F^{k}= \begin{cases}E^{k} & \text { if } \Gamma^{k} \text { is a forest, } \\ E^{k} \backslash\left(\cup_{\kappa \in \mathbf{C}\left(\Gamma^{k}\right)} E(\kappa)\right) & \text { if } \Gamma^{k} \text { is not a forest. }\end{cases}
$$

Note that $\Phi^{k}$ is a forest.

Next, we describe the set of essential coalitions with respect to $\left(N, c^{*}\right)$. With minor abuse of language, we write essential coalition when we refer to an essential coalition with respect to $\left(N, c^{*}\right)$. By Theorem 4.1, all essential coalitions are connected in every mest for $N$. Note that, since $F^{k}$ is contained in any mest for $N$, we have that every connected coalition in $F^{k}$ is also connected in any mcst for $N$. Next, we analyze those coalitions that are connected in every mcst for $N$ and that have no empty intersection with $\cup_{\kappa \in \mathbf{C}\left(\Gamma^{k}\right)} V(\kappa)$.

Lemma 4.2. Let $\left(N_{0}, C\right)$ be a 2-mcst network with $c_{i j} \in\{k, K\}, 0 \leq k \leq K$, for every $i, j \in N_{0}$ with $i \neq j$, and let $S \subset N$ with $S \in \mathcal{C}\left(\Gamma^{k}\right)$. If there exists $\kappa \in \mathbf{C}\left(\Gamma^{k}\right)$ with $1<|S \cap V(\kappa)|<|V(\kappa)|$, then, $S \notin \mathcal{C}\left(\hat{\Gamma}_{\mid N}\right)$ for at least one mcst $\hat{\Gamma}$ for $N$.

Proof: Let $\kappa \in \mathbf{C}\left(\Gamma^{k}\right)$ with $1<|S \cap V(\kappa)|<|V(\kappa)|$. Let $i, j \in S \cap V(\kappa)$ and let $k \in V(\kappa) \backslash S$. Then, there are two disjoint paths in $\kappa$ connecting $i$ and $j$, one of them visiting $k$. Let the edge $e$ be on the path that does not visit $k$. Using Kruskal's algorithm, we can find an mcst $\hat{\Gamma}$ containing all edges of $\kappa$ with the exception of $e$. The only path connecting $i$ and $j$ that uses only edges in $E(\hat{\Gamma})$ visits village $k$. Therefore, $S$ is not connected in $\hat{\Gamma}$.

As an immediate consequence of Theorem 4.1 and Lemma 4.2, we have the following result: if an essential coalition has nonempty intersection with the set of nodes of a cycle of the $k$-graph, then, the intersection is either a singleton, or the set of nodes of the cycle.

Corollary 4.3. Let $\left(N_{0}, C\right)$ be a 2-mcst network with $c_{i j} \in\{k, K\}, 0 \leq k \leq K$, for every $i, j \in N_{0}$ with $i \neq j$, let $(N, c)$ be the associated 2-mcst game, and let $S \in \operatorname{Ess}\left(c^{*}\right)$. If there exists $\kappa \in \mathbf{C}\left(\Gamma^{k}\right)$ with $S \cap V(\kappa) \neq \emptyset$, then, either $S \cap V(\kappa)=V(\kappa)$, or $S \cap V(\kappa)=\{i\}$ for some $i \in S$.

Given a 2-mcst network $\left(N_{0}, C\right)$ with $k$-graph $\Gamma^{k}$ and 2-mcst game $(N, c)$, as a result of Corollary 4.3 , we have that if two cycles $\kappa_{1}, \kappa_{2} \in \mathbf{C}\left(\Gamma^{k}\right)$ share two nodes, then, an essential coalition, $S$, of the dual game satisfies that either $\left|S \cap\left(V\left(\kappa_{1}\right) \cup V\left(\kappa_{2}\right)\right)\right| \leq 1$, or $V\left(\kappa_{1}\right) \cup V\left(\kappa_{2}\right) \subset S$. Following this, we define an equivalence relation in order to describe the set of essential coalitions of the dual game of a 2-mcst game.

Let $\Gamma=\left(N_{0}, E\right)$ be a graph and let $\kappa_{1}, \kappa_{2} \in \mathbf{C}(\Gamma)$. We say that $\kappa_{1}$ and $\kappa_{2}$ are related, $\kappa_{1} R \kappa_{2}$, if $\left|V\left(\kappa_{1}\right) \cap V\left(\kappa_{2}\right)\right| \geq 2$. Note that the binary relation $R$ is reflexive and symmetric, but does not need to 
be transitive. Therefore, we consider the transitive closure of $R, R^{+}$. It follows that $R^{+}$is an equivalence relation. We denote by $[\kappa]$ the equivalence class of $\kappa \in \mathbf{C}(\Gamma)$ with respect to $R^{+}$and by $[\mathbf{C}(\Gamma)]$ the set of equivalence classes in $\Gamma$, that is, $[\mathbf{C}(\Gamma)]=\{[\kappa]: \kappa \in \mathbf{C}(\Gamma)\}$. Given $\kappa \in \mathbf{C}(\Gamma)$, we denote $V([\kappa])=$ $\cup_{\tilde{\kappa} \in[\kappa]} V(\tilde{\kappa})$ and $E([\kappa])=\cup_{\tilde{\kappa} \in[\kappa]} E(\tilde{\kappa})$.

Let $\left(N_{0}, C\right)$ be a 2-mcst network. We define $\mathcal{E}\left(N_{0}, C\right)$ as follows,

$$
\mathcal{E}\left(N_{0}, C\right)=\left\{S \in \mathcal{C}\left(\Gamma^{k}\right):|S \cap V([\kappa])| \in\{0,1,|V([\kappa])|\} \text { for every }[\kappa] \in\left[\mathbf{C}\left(\Gamma^{k}\right)\right]\right\} .
$$

The following result is a direct consequence of Theorem 4.1 and Corollary 4.3 and the proof is, therefore, omitted.

Corollary 4.4. Let $\left(N_{0}, C\right)$ be a 2-mcst network with $c_{i j} \in\{k, K\}, 0 \leq k \leq K$, for every $i, j \in N_{0}$ with $i \neq j$, and let $(N, c)$ be the associated 2-mcst game. Then, $\operatorname{Ess}\left(c^{*}\right) \subset \mathcal{E}\left(N_{0}, C\right)$.

The following result states that if two essential coalitions have a nonempty intersection, then, the sum of their values in the dual of the 2-mcst game equals the sum of the values of their union and intersection in the dual of the 2-mcst game. Due to the technicality of the proof, this is postponed to the Appendix.

Lemma 4.5. Let $\left(N_{0}, C\right)$ be a 2-mcst network with $c_{i j} \in\{k, K\}, 0 \leq k \leq K$, for every $i, j \in N_{0}$ with $i \neq j$, and let $(N, c)$ be the associated 2-mcst game. Then,

$$
c^{*}\left(T_{1}\right)+c^{*}\left(T_{2}\right)=c^{*}\left(T_{1} \cap T_{2}\right)+c^{*}\left(T_{1} \cup T_{2}\right)
$$

for every $T_{1}, T_{2} \in \mathcal{E}\left(N_{0}, C\right)$ with $T_{1} \cap T_{2} \neq \emptyset$.

Next, we provide de main result of this Section.

Theorem 4.6. Let $\left(N_{0}, C\right)$ be a 2-mcst network with $c_{i j} \in\{k, K\}, 0 \leq k \leq K$, for every $i, j \in N_{0}$ with $i \neq j$, and let $(N, c)$ be the associated 2-mcst game. Let $b \in \mathbb{R}_{+}^{N}$ be a vector of revenues satisfying Assumption (3.1). Then, $\left(N, v_{b}\right)$ has a nonempty core.

Proof: By Theorem 3.2, it suffices to show that $\left(N, c^{*}\right)$ has a large core. By Theorem 2.1, we have to show that, for all $\mathcal{C} \in \operatorname{MC}(N), \sum_{T \in \mathcal{C}} c^{*}(T) \leq c^{*}(N)+\max _{\mu \in M(\mathcal{C})}\left\{\sum_{T \in 2^{E(\mathcal{C})} \backslash\{\emptyset\}} \mu_{T} c^{*}(T)\right\}$. Note that, as already mentioned in Section 2, we can restrict our analysis to minimal covers whose elements are essential coalitions of $\left(N, c^{*}\right)$. Therefore, fix $\mathcal{C} \in \mathbf{M C}(N)$ such that $T \in \operatorname{Ess}\left(c^{*}\right)$ for every $T \in \mathcal{C}$. By Corollary 4.4, $T \in \mathcal{E}\left(N_{0}, C\right)$ for every $T \in \mathcal{C}$. Let $\mathcal{C}=\left\{T_{1}, \ldots, T_{l_{1}}, \ldots, T_{l_{r-1}}, \ldots, T_{l_{r}}\right\}$ be ordered as follows: let $l_{0}=0$, then, $T_{l_{s-1}+1} \cap T_{l_{s-1}+2} \neq \emptyset, \ldots,\left(\cup_{l=l_{s-1}+1}^{l_{s}-1} T_{l}\right) \cap T_{l_{s}} \neq \emptyset$ for every $s \in\{1, \ldots, r\}$ and $\left(\cup_{l=l_{s-1}+1}^{l_{s}} T_{l}\right) \cap\left(\cup_{l=l_{s}+1}^{l_{r}} T_{l}\right)=\emptyset$ for every $s \in\{1, \ldots, r-1\}$. Note that $\left\{\cup_{l=1}^{l_{1}} T_{l}, \ldots, \cup_{l=l_{r-1}+1}^{l_{r}} T_{l}\right\}$ is a partition of $N$. Then,

$\sum_{l=1}^{l_{r}} c^{*}\left(T_{l}\right)=\sum_{l=1}^{l_{1}} c^{*}\left(T_{l}\right)+\sum_{l=l_{1}+1}^{l_{2}} c^{*}\left(T_{l}\right)+\ldots+\sum_{l=l_{r-1}+1}^{l_{r}} c^{*}\left(T_{l}\right)$ 


$$
\begin{aligned}
& =c^{*}\left(\cup_{l=1}^{l_{1}} T_{l}\right)+\sum_{l=1}^{l_{1}-1} c^{*}\left(\left(\cup_{\bar{l}=1}^{l} T_{\bar{l}}\right) \cap T_{l+1}\right)+\ldots+c^{*}\left(\cup_{l=l_{r-1}+1}^{l_{r}} T_{l}\right)+\sum_{l=l_{r-1}+1}^{l_{r}-1} c^{*}\left(\left(\cup_{\bar{l}=l_{r-1}+1}^{l} T_{\bar{l}}\right) \cap T_{l+1}\right) \\
& =\sum_{s=1}^{r} c^{*}\left(\cup_{l=l_{s-1}+1}^{l_{s}} T_{l}\right)+\sum_{s=1}^{r} \sum_{l=l_{s-1}+1}^{l_{s}-1} c^{*}\left(\left(\cup_{\bar{l}=l_{s-1}+1}^{l} T_{\bar{l}}\right) \cap T_{l+1}\right) \\
& \leq c^{*}(N)+\sum_{s=1}^{r} \sum_{l=l_{s-1}+1}^{l_{s}-1} c^{*}\left(\left(\cup_{\bar{l}=l_{s-1}+1}^{l} T_{\bar{l}}\right) \cap T_{l+1}\right) \\
& \leq c^{*}(N)+\max _{\mu \in M(\mathcal{C})}\left\{\sum_{T \in 2^{E(\mathcal{C})} \backslash\{\emptyset\}} \mu_{T} c^{*}(T)\right\},
\end{aligned}
$$

where the second equality is a consequence of successively applying Lemma 4.5; the first inequality follows because $\left\{\cup_{l=1}^{l_{1}} T_{l}, \ldots, \cup_{l=l_{r-1}+1}^{l_{r}} T_{l}\right\}$ is a partition of $N$ and $\left(N, c^{*}\right)$ is forest-essential with respect to any mcst $\Gamma$ for $N$ by Theorem 4.1; finally, the last inequality follows because $\mu$ defined as $\mu_{T}=1$ if $T=\left(\cup_{\bar{l}=l_{s-1}+1}^{l} T_{\bar{l}}\right) \cap T_{l+1}$ for some $s \in\{1, \ldots, r\}$ and $l \in\left\{l_{s-1}+1, \ldots, l_{s}-1\right\}$ and $\mu_{T}=0$ otherwise, is an mc-balanced vector for $\mathcal{C}$.

To conclude this Section, note that the cost game in Example 3.1 is a 2 -mcst game with $k=1$, $K=2$, and $C$ defined as $c_{i j}=1$ if $\{i, j\} \in\{\{0,4\},\{0,5\},\{0,6\},\{1,4\},\{1,5\},\{2,4\},\{2,6\},\{3,5\},\{3,6\}\}$ and $c_{i, j}=2$ otherwise. Therefore, Assumption (3.1) it is, indeed, necessary in Theorem 4.6.

\section{$5 \quad$ Further remarks}

Littlechild and Owen (1976) were the first to analyze cost problems arising from a general service facility by taking into account the profits that the service generates. They restricted their study to the framework of airport problems, with a more recent follow up by Brânzei et al. (2006). Meertens and Potters (2006) consider fixed tree games with revenues. In both settings, the underlying cost game is concave ${ }^{6}$ and, therefore, its dual game is convex ${ }^{7}$ and has a large core (see Sharkey 1982). Estévez-Fernández et al. (2009) consider routing games with revenues. A routing game is similar to a traveling salesman game (see Potters et al. 1992), except that a proper coalition cannot choose the order of the visits of the salesman, but must use the order induced by an optimal order to visit all cities ${ }^{8}$. Example 5.1 shows that the dual of a routing game does not always have a large core.

\footnotetext{
${ }^{6} \mathrm{~A}$ cost game $(N, c)$ is concave if, for every $i \in N$ and every $S \subset T \subset N \backslash\{i\}, c(S \cup\{i\})-c(S) \geq c(T \cup\{i\})-c(T)$.

${ }^{7} \mathrm{~A}$ game $(N, v)$ is convex if, for every $i \in N$ and every $S \subset T \subset N \backslash\{i\}, v(S \cup\{i\})-v(S) \leq v(T \cup\{i\})-v(T)$.

${ }^{8}$ The cities are usually numbered in such a way that this is the increasing order.
} 
Example 5.1. Consider the routing problem with revenues $\left(N_{0}, C, b\right)$ with $N=\{1,2,3,4,5\}$,

$$
\begin{aligned}
& \mathbf{0} \\
& \mathbf{1} \\
& \mathbf{2} \\
& \mathbf{3} \\
& \mathbf{4} \\
& \mathbf{5}
\end{aligned}\left(\begin{array}{cccccc}
0 & \mathbf{1} & \mathbf{2} & \mathbf{3} & \mathbf{4} & \mathbf{5} \\
0 & 2 & 1 & 10 & 2 & 10 \\
2 & 0 & 2 & 12 & 1 & 12 \\
1 & 2 & 0 & 10 & 2 & 11 \\
10 & 12 & 10 & 0 & 2 & 20 \\
2 & 1 & 2 & 2 & 0 & 2 \\
10 & 12 & 11 & 20 & 2 & 0
\end{array}\right)=C
$$

$b=(10,10,10.5,10,10)$, and optimal tour $\hat{\pi}: 0-1-2-3-4-5-0$. In Table 4 , we provide the coalitional

\begin{tabular}{|c|c|c|c|c|c|c|c|c|c|c|c|c|c|c|c|}
\hline$S$ & $\{1\}$ & $\{2\}$ & $\{3\}$ & $\{4\}$ & $\{5\}$ & $\{1,2\}$ & $\{1,3\}$ & $\{1,4\}$ & $\{1,5\}$ & $\{2,3\}$ & $\{2,4\}$ & $\{2,5\}$ & $\{3,4\}$ & $\{3,5\}$ & $\{4,5\}$ \\
\hline$c_{\hat{\pi}}(S)$ & 4 & 2 & 20 & 4 & 20 & 5 & 24 & 5 & 24 & 21 & 5 & 22 & 14 & 40 & 14 \\
\hline$c_{\hat{\pi}}^{*}(S)$ & 3 & 0 & 10 & -16 & 10 & 4 & 13 & -1 & 13 & 13 & -16 & 10 & 3 & 20 & 4 \\
\hline$b(S)$ & 10 & 10 & 10.5 & 10 & 10 & 20 & 20.5 & 20 & 20 & 20.5 & 20 & 20 & 20.5 & 20.5 & 20 \\
\hline$v_{\hat{\pi} b}(S)$ & 6 & 8 & 0 & 6 & 0 & 15 & 6 & 15 & 6 & 8 & 15 & 8 & \multicolumn{2}{|l|}{6.5} & 6 \\
\hline$S$ & \multicolumn{2}{|c|}{$1,2,3$} & $\{1,2,4\}$ & \multicolumn{2}{|c|}{$1,2,5$} & \multicolumn{2}{|c|}{$1,3,4$} & $\{1,3,5\}$ & $\{1,4,5\}$ & $\{2,3,4\}$ & $\{2,3,5\}$ & \multicolumn{2}{|c|}{$2,4,5$} & $\{3,4,5\}$ & \\
\hline$c_{\hat{\pi}}(S)$ & \multicolumn{2}{|c|}{24} & 8 & \multicolumn{2}{|c|}{25} & 18 & \multicolumn{2}{|c|}{44} & 15 & 15 & 41 & \multicolumn{2}{|c|}{15} & 24 & \\
\hline$c_{\hat{\pi}}^{*}(S)$ & \multicolumn{2}{|c|}{14} & -12 & \multicolumn{2}{|r|}{14} & \multicolumn{2}{|l|}{6} & 23 & 7 & 4 & 23 & \multicolumn{2}{|c|}{4} & 23 & \\
\hline$b(S)$ & \multicolumn{2}{|c|}{30.5} & 30 & \multicolumn{2}{|r|}{30} & 30.5 & \multicolumn{2}{|c|}{30.5} & 30 & 30.5 & 30.5 & & 30 & 30.5 & \\
\hline$v_{\hat{\pi} b}(S)$ & 15 & & 22 & & 15 & 15 & 6 & 3 & 15 & 15.5 & 8 & & 15 & 6.5 & \\
\hline$S$ & $\{1,2$, & $3,4\}$ & $\{1,2$, & $, 3,5\}$ & $\{1,2$ & $2,4,5\}$ & $\{1,3,4$, & & $\{2,3,4,5\}$ & $N$ & & & & & \\
\hline$c_{\hat{\pi}}(S)$ & 1 & 8 & 4 & 4 & & 18 & 28 & & 25 & 28 & & & & & \\
\hline$c_{\hat{\pi}}^{*}(S)$ & 8 & 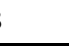 & 2 & 4 & & 8 & 26 & & 24 & 28 & & & & & \\
\hline$b(S)$ & 40 & & 40 & .5 & & 40 & 40.5 & & 40.5 & 50.5 & & & & & \\
\hline$v_{\hat{\pi} b}(S)$ & 22 & & 1 & 5 & & 22 & 15 & & 15.5 & 22.5 & & & & & \\
\hline
\end{tabular}
values of the routing game $\left(N, c_{\hat{\pi}}\right)$, the corresponding dual game $\left(N, c_{\hat{\pi}}^{*}\right),(N, b)$ and $\left(N, v_{\hat{\pi} b}\right)$.

Table 4: Coalitional values of the routing game, associated dual game, additive revenues, and routing game with revenues in Example 5.1.

Note that $b(S) \geq c^{*}(S)$ for every $S \subset N$. Still, $\left(N, v_{\hat{\pi} b}\right)$ has an empty core. To show this, suppose that $x \in \operatorname{Core}\left(v_{\hat{\pi} b}\right)$; then, $15 \leq x(\{1,4\}), 8 \leq x_{2}$, and $0 \leq x(\{3,5\})$. By adding all these equations we obtain $23 \leq x(N)=v_{\hat{\pi} b}(N)=22.5$ establishing a contradiction. Therefore, Core $\left(v_{\hat{\pi} b}\right)=\emptyset$.

Hence, to ensure balancedness, Assumption (3.1) is insufficient. Estévez-Fernández et al. (2009) show that balancedness is guaranteed if in the corresponding traveling salesman problem with revenues, it is optimal for $N$ to visit all cities and to visit them in increasing order. This boils down to $b(S) \geq c_{t s p}^{*}(S)$ for every $S \subset N$, where $\left(N, c_{t s p}\right)$ denotes the traveling salesman game corresponding to distance matrix $\mathrm{C}$. 
In Table 5 , we provide the coalitional values of the traveling salesman game $\left(N, c_{t s p}\right)$, the corresponding dual game $\left(N, c_{t s p}^{*}\right)$, and $(N, b)$. Note that it is more profitable to only visit cities $\{1,2,3,4\}$ than to visit all cities in $N$. Besides, note that $b$ is not an element of the upper core of $\left(N, c_{t s p}^{*}\right)$ since $b_{3}=10.5<11=$ $c_{t s p}^{*}(\{3\})$ and $b_{5}=10<12=c_{t s p}^{*}(\{5\})$.

\begin{tabular}{|c|c|c|c|c|c|c|c|c|c|c|c|c|c|c|c|}
\hline$S$ & $\{1\}$ & $\{2\}$ & $\{3\}$ & $\{4\}$ & $\{5\}$ & $\{1,2\}$ & $\{1,3\}$ & $\{1,4$ & $\{1,5\}$ & $\{2,3\}$ & $\{2,4\}$ & $\{2,5\}$ & $\{3,4\}$ & $\{3,5\}$ & $\{4$, \\
\hline$c_{t s p}(S)$ & 4 & 2 & 20 & 4 & 20 & 5 & 24 & 5 & 24 & 21 & 5 & 22 & 14 & 40 & 14 \\
\hline$c_{t s p}^{*}(S)$ & 3 & 0 & 11 & -16 & 12 & 4 & 13 & -13 & 13 & 13 & -16 & 13 & 3 & 20 & 4 \\
\hline$b(S)$ & 10 & 10 & 10.5 & 10 & 10 & 20 & 20.5 & 20 & 20 & 20.5 & 20 & 20 & 20.5 & 20.5 & 20 \\
\hline$b(S)-c_{t s p}(S)$ & 6 & 8 & -9.5 & 6 & -10 & 15 & -3.5 & 15 & -4 & -0.5 & \multicolumn{2}{|l|}{15} & 6.5 & -19.5 & 6 \\
\hline$S$ & \multicolumn{2}{|c|}{$1,2,3$} & $\{1,2,4\}$ & \multicolumn{2}{|c|}{$1,2,5$} & \multicolumn{2}{|c|}{$1,3,4$} & $\{1,3,5\}$ & $\{1,4,5\}$ & $\{2,3,4\}$ & & $\{2,3,5\}$ & $\{2,4,5\}$ & \multicolumn{2}{|c|}{$3,4,5$} \\
\hline$c_{t s p}(S)$ & \multicolumn{2}{|c|}{24} & 8 & \multicolumn{2}{|r|}{25} & \multicolumn{2}{|c|}{15} & 44 & 15 & 15 & & 41 & 15 & \multicolumn{2}{|c|}{24} \\
\hline$c_{t s p}^{*}(S)$ & \multicolumn{2}{|c|}{14} & -12 & \multicolumn{2}{|r|}{14} & \multicolumn{2}{|l|}{6} & 23 & 7 & 4 & & 23 & 4 & \multicolumn{2}{|l|}{23} \\
\hline$b(S)$ & \multicolumn{2}{|c|}{30.5} & 30 & \multicolumn{2}{|r|}{30} & \multicolumn{2}{|c|}{30.5} & 30.5 & 30 & 30.5 & & 30.5 & 30 & 30.5 & \\
\hline$b(S)-c_{t s p}(S)$ & 6.5 & & 22 & & 5 & 15. & & -13.5 & 15 & 15.5 & & -10.5 & 15 & 6.5 & \\
\hline$S$ & $\{1,2$, & $3,4\}$ & $\{1,2$, & $3,5\}$ & $\{1,2$ & $2,4,5\}$ & $\{1,3$ & $, 4,5\}$ & $\{2,3,4,5\}$ & $N$ & & & & & \\
\hline$c_{t s p}(S)$ & 1 & & 4 & 4 & & 17 & & 28 & 25 & 28 & & & & & \\
\hline$c_{t s p}^{*}(S)$ & 8 & & 2 & 4 & & 8 & & 26 & 24 & 28 & & & & & \\
\hline$b(S)$ & 40 & & 40 & .5 & & 40 & & 0.5 & 40.5 & 50.5 & & & & & \\
\hline$b(S)-c_{t s p}(S)$ & 24 & & -3 & 3.5 & & 23 & & 2.5 & 15.5 & 22.5 & & & & & \\
\hline
\end{tabular}

Table 5: Coalitional values of the traveling salesman game, associated dual game, additive revenues, and net revenues in Example 5.1.

\section{Appendix}

In this Appendix, we show the proof of Lemma 4.5 in Section 4. Before giving the proof, we provide three auxiliary lemmas.

Lemma A.1. Let $\left(N_{0}, C\right)$ be a 2-mcst network with $c_{i j} \in\{k, K\}, 0 \leq k \leq K$, for every $i, j \in N_{0}$ with $i \neq j$, and let $(N, c)$ be the corresponding 2 -mcst game. Then,

$c^{*}(T)=|T| k+(k-K)\left(\sum_{i \in T}\left(\left|\left\{j \in N_{0} \backslash T:\{i, j\} \in F^{k}\right\}\right|+\left|\left\{[\kappa] \in\left[\mathbf{C}\left(\Gamma^{k}\right)\right]: V([\kappa]) \cap T=\{i\}\right\}\right|\right)-1\right)$

for every $T \in \mathcal{E}\left(N_{0}, C\right)$. 
Proof: By definition of $\mathcal{E}\left(N_{0}, C\right)$, we have that $T \in \mathcal{C}\left(\Gamma^{k}\right)$ and, therefore, there exists $U \in N_{0 \mid \Gamma^{k}}$ with $T \subset U$. Then,

$$
\begin{aligned}
c^{*}(T)= & c(N)-c(N \backslash T) \\
= & k|N|-k\left(\left|\Gamma_{\mid N_{0}}^{k}\right|-1\right)+K\left(\left|\Gamma_{\left|N_{0}\right|}^{k}\right|-1\right) \\
& -\left(k|N \backslash T|-k\left(\left|\Gamma_{\mid\left(N_{0} \backslash T\right)}^{k}\right|-1\right)+K\left(\left|\Gamma_{\mid\left(N_{0} \backslash T\right)}^{k}\right|-1\right)\right) \\
= & k|T|-(K-k)\left(\left|\Gamma_{\mid\left(N_{0} \backslash T\right)}^{k}\right|-\left|\Gamma_{\mid N_{0}}^{k}\right|\right) \\
= & k|T|-(K-k)\left(\left|\Gamma_{\mid(U \backslash T)}^{k}\right|-1\right) \\
= & k|T| \\
& -(K-k)\left(\sum_{i \in T}\left(\left|\left\{j \in U \backslash T:\{i, j\} \in F^{k}\right\}\right|+\left|\left\{[\kappa] \in\left[\mathbf{C}\left(\Gamma^{k}\right)\right]: V([\kappa]) \cap T=\{i\}\right\}\right|\right)-1\right) \\
= & k|T|- \\
& (K-k)\left(\sum_{i \in T}\left(\left|\left\{j \in N_{0} \backslash T:\{i, j\} \in F^{k}\right\}\right|+\left|\left\{[\kappa] \in\left[\mathbf{C}\left(\Gamma^{k}\right)\right]: V([\kappa]) \cap T=\{i\}\right\}\right|\right)-1\right),
\end{aligned}
$$

where the fourth equality is a direct consequence of $T \subset U$ with $U \in N_{0 \mid \Gamma^{k}}$; the fifth equality follows because the elements of $\Gamma_{\mid(U \backslash T)}^{k}$ are those components that we obtain when the edges between $T$ and $U \backslash T$ are broken; the sixth equality is a direct consequence of $T \subset U$ and, then, $\{i, j\} \subset U$ for every $\{i, j\} \in E^{k}$ with $i \in T$.

Lemma A.2. Let $\left(N_{0}, C\right)$ be a 2-mcst network with $c_{i j} \in\{k, K\}, 0 \leq k \leq K$, for every $i, j \in N_{0}$ with $i \neq j$. If $T_{1}, T_{2} \in \mathcal{E}\left(N_{0}, C\right)$ with $T_{1} \cap T_{2} \neq \emptyset$. Then,

(i) If $\left|V([\kappa]) \cap T_{1}\right|=\left|V([\kappa]) \cap T_{2}\right|=1$ for some $[\kappa] \in\left[\mathbf{C}\left(\Gamma^{k}\right)\right]$, then, $V([\kappa]) \cap T_{1}=V([\kappa]) \cap T_{2}$;

(ii) $T_{1} \cup T_{2}, T_{1} \cap T_{2} \in \mathcal{E}\left(N_{0}, C\right)$.

\section{Proof:}

(i) On the contrary, suppose that $V([\kappa]) \cap T_{1}=\left\{i_{1}\right\}$ and $V([\kappa]) \cap T_{2}=\left\{i_{2}\right\}$ with $i_{1} \neq i_{2}$. Since $\left|V([\kappa]) \cap T_{1}\right|=1$ and $\left|V([\kappa]) \cap T_{2}\right|=1$, we have that $i_{1} \in T_{1} \backslash T_{2}$ and $i_{2} \in T_{2} \backslash T_{1}$. Note that $V([\kappa]) \in \mathcal{C}\left(\Gamma^{k}\right)$ and, therefore, there exists a path connecting $i_{1}$ and $i_{2}, \operatorname{path}\left(i_{1}, i_{2}\right)$, using only edges in $E([\kappa])$.

Let $j \in T_{1} \cap T_{2}$; by connectivity of $T_{1}$, there exists a path, $\operatorname{path}\left(i_{1}, j\right)$, connecting $i_{1}$ and $j$ in $\Gamma^{k}$ using nodes of $T_{1}$. Note that no edge of $\operatorname{path}\left(i_{1}, j\right)$ belongs to $E([\kappa])$ since $\left|V([\kappa]) \cap T_{1}\right|=1$. 
Analogously, there exists a path, $\operatorname{path}\left(j, i_{2}\right)$, connecting $j$ and $i_{2}$ in $\Gamma^{k}$ using nodes of $T_{2}$ and such that, analogously as before, no edge in the path belongs to $E([\kappa])$. Therefore, combining $\operatorname{path}\left(i_{1}, j\right)$ and $\operatorname{path}\left(j, i_{2}\right)$, we can construct a path connecting $i_{1}$ and $i_{2}, \operatorname{path}\left(i_{1}, i_{2}\right)^{*}$, without using any edge in $E([\kappa])$. Combining the paths $\operatorname{path}\left(i_{1}, i_{2}\right)$ and $\operatorname{path}\left(i_{1}, i_{2}\right)^{*}$, there exists a cycle $\bar{\kappa} \in \mathbf{C}\left(\Gamma^{k}\right)$ with $i_{1}, i_{2} \in V([\bar{\kappa}])$ and $[\kappa] \neq[\bar{\kappa}]$. This establishes a contradiction since $i_{1}, i_{2} \in V([\kappa])$ and, therefore, $[\kappa]=[\bar{\kappa}]$.

(ii) First, note that $T_{1} \cup T_{2} \in \mathcal{C}\left(\Gamma^{k}\right)$ since $T_{1}, T_{2} \in \mathcal{C}\left(\Gamma^{k}\right)$ with $T_{1} \cap T_{2} \neq \emptyset$. Moreover, for every $[\kappa] \in\left[\mathbf{C}\left(\Gamma^{k}\right)\right]$, we have that

a. if $V([\kappa]) \subset T_{1}$ (analogously, $V([\kappa]) \subset T_{2}$ ), then, $V([\kappa]) \subset T_{1} \cup T_{2}$;

b. if $V([\kappa]) \cap T_{1}=\{i\}$ for some $i \in T_{1}$ and $\left|V([\kappa]) \cap T_{2}\right|=1$ (analogously, $V([\kappa]) \cap T_{2}=\{i\}$ for some $i \in T_{2}$ and $\left|V([\kappa]) \cap T_{1}\right|=1$ ), then, $V([\kappa]) \cap T_{2}=\{i\}$ by (i) of this Lemma and $V([\kappa]) \cap\left(T_{1} \cup T_{2}\right)=\{i\} ;$

c. if $V([\kappa]) \cap T_{1}=\{i\}$ for some $i \in T_{1}$ and $V([\kappa]) \cap T_{2}=\emptyset$ (analogously, $V([\kappa]) \cap T_{2}=\{i\}$ for some $i \in T_{2}$ and $\left.V([\kappa]) \cap T_{1}=\emptyset\right)$, then, $V([\kappa]) \cap\left(T_{1} \cup T_{2}\right)=\{i\}$;

d. if $V([\kappa]) \cap T_{1}=\emptyset$ and $V([\kappa]) \cap T_{2}=\emptyset$, then, $V([\kappa]) \cap\left(T_{1} \cup T_{2}\right)=\emptyset$.

Therefore, $T_{1} \cup T_{2} \in \mathcal{E}\left(N_{0}, C\right)$.

Second, note that the intersection of two connected sets is connected and, therefore, $T_{1} \cap T_{2} \in \mathcal{C}\left(\Gamma^{k}\right)$.

Moreover, for every $[\kappa] \in\left[\mathbf{C}\left(\Gamma^{k}\right)\right]$, we have that

e. if $V([\kappa]) \cap T_{1}=\emptyset$ (analogously, $V([\kappa]) \cap T_{2}=\emptyset$ ), then, $V([\kappa]) \cap\left(T_{1} \cap T_{2}\right)=\emptyset$;

f. if $V([\kappa]) \cap T_{1}=\{i\}$ for some $i \in T_{1}$ and $\left|V([\kappa]) \cap T_{2}\right|=1$ (analogously, $V([\kappa]) \cap T_{2}=\{i\}$ for some $i \in T_{2}$ and $\left|V([\kappa]) \cap T_{1}\right|=1$ ), then, $V([\kappa]) \cap T_{2}=\{i\}$ by (i) of this Lemma and $V([\kappa]) \cap\left(T_{1} \cap T_{2}\right)=\{i\} ;$

g. if $V([\kappa]) \cap T_{1}=\{i\}$ for some $i \in T_{1}$ and $V([\kappa]) \subset T_{2}$ (analogously, $V([\kappa]) \cap T_{2}=\{i\}$ for some $i \in T_{2}$ and $\left.V([\kappa]) \subset T_{1}\right)$, then, $V([\kappa]) \cap\left(T_{1} \cap T_{2}\right)=\{i\} ;$

h. if $V([\kappa]) \subset T_{1}$ and $V([\kappa]) \subset T_{2}$, then, $V([\kappa]) \subset T_{1} \cap T_{2}$.

Therefore, $T_{1} \cap T_{2} \in \mathcal{E}\left(N_{0}, C\right)$.

Lemma A.3. Let $\left(N_{0}, C\right)$ be a 2-mcst network with $c_{i j} \in\{k, K\}, 0 \leq k \leq K$, for every $i, j \in N_{0}$ with $i \neq j$, and let $T_{1}, T_{2} \in \mathcal{E}\left(N_{0}, C\right)$ with $T_{1} \cap T_{2} \neq \emptyset$. Then,

(i) $\sum_{i \in T_{1} \backslash T_{2}}\left|\left\{j \in T_{2} \backslash T_{1}:\{i, j\} \in F^{k}\right\}\right|+\sum_{i \in T_{2} \backslash T_{1}}\left|\left\{j \in T_{1} \backslash T_{2}:\{i, j\} \in F^{k}\right\}\right|=0$; 
(ii) $\sum_{i \in T_{1}}\left|\left\{j \in N_{0} \backslash T_{1}:\{i, j\} \in F^{k}\right\}\right|+\sum_{i \in T_{2}}\left|\left\{j \in N_{0} \backslash T_{2}:\{i, j\} \in F^{k}\right\}\right|=$

$\sum_{i \in T_{1} \cup T_{2}}\left|\left\{j \in N_{0} \backslash\left(T_{1} \cup T_{2}\right):\{i, j\} \in F^{k}\right\}\right|+\sum_{i \in T_{1} \cap T_{2}}\left|\left\{j \in N_{0} \backslash\left(T_{1} \cap T_{2}\right):\{i, j\} \in F^{k}\right\}\right| ;$

(iii) $\sum_{i \in T_{1}}\left|\left\{[\kappa] \in\left[\mathbf{C}\left(\Gamma^{k}\right)\right]: V([\kappa]) \cap T_{1}=\{i\}\right\}\right|+\sum_{i \in T_{2}}\left|\left\{[\kappa] \in\left[\mathbf{C}\left(\Gamma^{k}\right)\right]: V([\kappa]) \cap T_{2}=\{i\}\right\}\right|=$

$\sum_{i \in T_{1} \cup T_{2}}\left|\left\{[\kappa] \in\left[\mathbf{C}\left(\Gamma^{k}\right)\right]: V([\kappa]) \cap\left(T_{1} \cup T_{2}\right)=\{i\}\right\}\right|$

$+\sum_{i \in T_{1} \cap T_{2}}\left|\left\{[\kappa] \in\left[\mathbf{C}\left(\Gamma^{k}\right)\right]: V([\kappa]) \cap\left(T_{1} \cap T_{2}\right)=\{i\}\right\}\right|$.

Proof: First, note that $T_{1} \cup T_{2}, T_{1} \cap T_{2} \in \mathcal{E}\left(N_{0}, C\right)$ by Lemma A.2 (ii).

(i) On the contrary, suppose that

$$
\sum_{i \in T_{1} \backslash T_{2}}\left|\left\{j \in T_{2} \backslash T_{1}:\{i, j\} \in F^{k}\right\}\right|+\sum_{i \in T_{2} \backslash T_{1}}\left|\left\{j \in T_{1} \backslash T_{2}:\{i, j\} \in F^{k}\right\}\right| \neq 0 .
$$

Then, there exist $i_{1} \in T_{1} \backslash T_{2}$ and $i_{2} \in T_{2} \backslash T_{1}$ with $\left\{i_{1}, i_{2}\right\} \in F^{k}$. Note that, by definition of $F^{k},\left\{i_{1}, i_{2}\right\} \notin \cup_{\kappa \in \mathcal{C}\left(\Gamma^{k}\right)} E(\kappa)$. Let $j \in T_{1} \cap T_{2}$; by connectivity of $T_{1}$, there exists a path, $\operatorname{path}\left(i_{1}, j\right)$, connecting $i_{1}$ and $j$ in $\Gamma^{k}$ using nodes of $T_{1}$. Analogously, there exists a path, $\operatorname{path}\left(j, i_{2}\right)$, connecting $j$ and $i_{2}$ in $\Gamma^{k}$ using nodes of $T_{2}$. Therefore, combining $\operatorname{path}\left(i_{1}, j\right)$ and $\operatorname{path}\left(j, i_{2}\right)$, we can construct a path, path $\left(i_{1}, i_{2}\right)$, connecting $i_{1}$ and $i_{2}$ without using the edge $\left\{i_{1}, i_{2}\right\}$. As a result, there exists a cycle $\kappa \in \mathbf{C}\left(\Gamma^{k}\right)$ with $\left\{i_{1}, i_{2}\right\} \in E(\kappa)$, establishing a contradiction.

(ii)

$$
\begin{aligned}
& \sum_{i \in T_{1}}\left|\left\{j \in N_{0} \backslash T_{1}:\{i, j\} \in F^{k}\right\}\right|+\sum_{i \in T_{2}}\left|\left\{j \in N_{0} \backslash T_{2}:\{i, j\} \in F^{k}\right\}\right| \\
&=\sum_{i \in T_{1} \backslash T_{2}}\left|\left\{j \in N_{0} \backslash T_{1}:\{i, j\} \in F^{k}\right\}\right|+\sum_{i \in T_{1} \cap T_{2}}\left|\left\{j \in N_{0} \backslash T_{1}:\{i, j\} \in F^{k}\right\}\right| \\
&+\sum_{i \in T_{2} \backslash T_{1}}\left|\left\{j \in N_{0} \backslash T_{2}:\{i, j\} \in F^{k}\right\}\right|+\sum_{i \in T_{1} \cap T_{2}}\left|\left\{j \in N_{0} \backslash T_{2}:\{i, j\} \in F^{k}\right\}\right| \\
&=\sum_{i \in T_{1} \backslash T_{2}}\left(\left|\left\{j \in N_{0} \backslash\left(T_{1} \cup T_{2}\right):\{i, j\} \in F^{k}\right\}\right|+\left|\left\{j \in T_{2} \backslash T_{1}:\{i, j\} \in F^{k}\right\}\right|\right) \\
&+\sum_{i \in T_{1} \cap T_{2}}\left(\left|\left\{j \in N_{0} \backslash\left(T_{1} \cup T_{2}\right):\{i, j\} \in F^{k}\right\}\right|+\left|\left\{j \in T_{2} \backslash T_{1}:\{i, j\} \in F^{k}\right\}\right|\right) \\
&+\sum_{i \in T_{2} \backslash T_{1}}\left(\left|\left\{j \in N_{0} \backslash\left(T_{1} \cup T_{2}\right):\{i, j\} \in F^{k}\right\}\right|+\left|\left\{j \in T_{1} \backslash T_{2}:\{i, j\} \in F^{k}\right\}\right|\right) \\
&+\sum_{i \in T_{1} \cap T_{2}}\left(\left|\left\{j \in N_{0} \backslash\left(T_{1} \cup T_{2}\right):\{i, j\} \in F^{k}\right\}\right|+\left|\left\{j \in T_{1} \backslash T_{2}:\{i, j\} \in F^{k}\right\}\right|\right) \\
&= \sum_{i \in T_{1} \cup T_{2}}\left|\left\{j \in N_{0} \backslash\left(T_{1} \cup T_{2}\right):\{i, j\} \in F^{k}\right\}\right|+\sum_{i \in T_{1} \backslash T_{2}}\left|\left\{j \in T_{2} \backslash T_{1}:\{i, j\} \in F^{k}\right\}\right| \\
&+\sum_{i \in T_{1} \cap T_{2}}\left|\left\{j \in T_{2} \backslash T_{1}:\{i, j\} \in F^{k}\right\}\right|+\sum_{i \in T_{2} \backslash T_{1}}\left|\left\{j \in T_{1} \backslash T_{2}:\{i, j\} \in F^{k}\right\}\right| \\
&+\sum_{i \in T_{1} \cap T_{2}}\left(\left|\left\{j \in N_{0} \backslash\left(T_{1} \cup T_{2}\right):\{i, j\} \in F^{k}\right\}\right|+\left|\left\{j \in T_{1} \backslash T_{2}:\{i, j\} \in F^{k}\right\}\right|\right)
\end{aligned}
$$




$$
\begin{aligned}
= & \sum_{i \in T_{1} \cup T_{2}}\left|\left\{j \in N_{0} \backslash\left(T_{1} \cup T_{2}\right):\{i, j\} \in F^{k}\right\}\right|+\sum_{i \in T_{1} \cap T_{2}}\left|\left\{j \in T_{2} \backslash T_{1}:\{i, j\} \in F^{k}\right\}\right| \\
& +\sum_{i \in T_{1} \cap T_{2}}\left(\left|\left\{j \in N_{0} \backslash\left(T_{1} \cup T_{2}\right):\{i, j\} \in F^{k}\right\}\right|+\left|\left\{j \in T_{1} \backslash T_{2}:\{i, j\} \in F^{k}\right\}\right|\right) \\
= & \sum_{i \in T_{1} \cup T_{2}}\left|\left\{j \in N_{0} \backslash\left(T_{1} \cup T_{2}\right):\{i, j\} \in F^{k}\right\}\right|+\sum_{i \in T_{1} \cap T_{2}}\left|\left\{j \in N_{0} \backslash\left(T_{1} \cap T_{2}\right):\{i, j\} \in F^{k}\right\}\right|,
\end{aligned}
$$

where the first equality follows because $T_{1}=\left(T_{1} \backslash T_{2}\right) \cup\left(T_{1} \cap T_{2}\right)$ and $T_{2}=\left(T_{2} \backslash T_{1}\right) \cup\left(T_{1} \cap T_{2}\right)$. The second equality is a direct consequence of $N_{0} \backslash T_{1}=\left(N_{0} \backslash\left(T_{1} \cup T_{2}\right)\right) \cup\left(T_{2} \backslash T_{1}\right)$ and $N_{0} \backslash T_{2}=$ $\left(N_{0} \backslash\left(T_{1} \cup T_{2}\right)\right) \cup\left(T_{1} \backslash T_{2}\right)$. The third equality follows since $T_{1} \cup T_{2}=\left(T_{1} \backslash T_{2}\right) \cup\left(T_{2} \backslash T_{1}\right) \cup\left(T_{1} \cap T_{2}\right)$. The fourth equality is a directed consequence of (i) of this Lemma. The fifth equality follows because $N_{0} \backslash\left(T_{1} \cap T_{2}\right)=\left(T_{2} \backslash T_{1}\right) \cup\left(N_{0} \backslash\left(T_{1} \cup T_{2}\right)\right) \cup\left(T_{1} \backslash T_{2}\right)$.

(iii) By definition of $\mathcal{E}\left(N_{0}, C\right)$, if $R \in \mathcal{E}\left(N_{0}, C\right)$ and $[\kappa] \in\left[\mathbf{C}\left(\Gamma^{k}\right)\right]$ with $V([\kappa]) \cap R \neq \emptyset$, then, either $V([\kappa]) \cap R=V([\kappa])$, or $V([\kappa]) \cap R=\{i\}$ for some $i \in R$. As a result, we have that

$$
\sum_{i \in R}\left|\left\{[\kappa] \in\left[\mathbf{C}\left(\Gamma^{k}\right)\right]: V([\kappa]) \cap R=\{i\}\right\}\right|=\left|\left\{[\kappa] \in\left[\mathbf{C}\left(\Gamma^{k}\right)\right]:|V([\kappa]) \cap R|=1\right\}\right| .
$$

For $R \subset N$, we denote $\left[\mathbf{C}\left(\Gamma^{k}\right)\right](R)=\left\{[\kappa] \in\left[\mathbf{C}\left(\Gamma^{k}\right)\right]:|V([\kappa]) \cap R|=1\right\}$. Next, we study the relation between the sets $\left[\mathbf{C}\left(\Gamma^{k}\right)\right]\left(T_{1}\right)$ and $\left[\mathbf{C}\left(\Gamma^{k}\right)\right]\left(T_{2}\right)$ and the sets $\left[\mathbf{C}\left(\Gamma^{k}\right)\right]\left(T_{1} \cup T_{2}\right)$ and $\left[\mathbf{C}\left(\Gamma^{k}\right)\right]\left(T_{1} \cap T_{2}\right)$. For this, we distinguish several cases:

a. $[\kappa] \in\left[\mathbf{C}\left(\Gamma^{k}\right)\right]\left(T_{1}\right)$ and $[\kappa] \in\left[\mathbf{C}\left(\Gamma^{k}\right)\right]\left(T_{2}\right)$. By Lemma A.2 (i), $V([\kappa]) \cap T_{1}=V([\kappa]) \cap T_{2}=\{i\}$ for some $i \in T_{1} \cap T_{2}$ and, then, $V([\kappa]) \cap\left(T_{1} \cup T_{2}\right)=\{i\}$ and $V([\kappa]) \cap\left(T_{1} \cap T_{2}\right)=\{i\}$. Therefore, $[\kappa] \in\left[\mathbf{C}\left(\Gamma^{k}\right)\right]\left(T_{1} \cup T_{2}\right)$ and $[\kappa] \in\left[\mathbf{C}\left(\Gamma^{k}\right)\right]\left(T_{1} \cap T_{2}\right)$.

b. $[\kappa] \in\left[\mathbf{C}\left(\Gamma^{k}\right)\right]\left(T_{1}\right)$ and $[\kappa] \notin\left[\mathbf{C}\left(\Gamma^{k}\right)\right]\left(T_{2}\right)$. In this case, we have to distinguish between two new cases: $V([\kappa]) \cap T_{2}=\emptyset$ and $V([\kappa]) \cap T_{2}=V([\kappa])$.

b.1. $V([\kappa]) \cap T_{2}=\emptyset$. In this case, $[\kappa] \in\left[\mathbf{C}\left(\Gamma^{k}\right)\right]\left(T_{1} \cup T_{2}\right)$ and $[\kappa] \notin\left[\mathbf{C}\left(\Gamma^{k}\right)\right]\left(T_{1} \cap T_{2}\right)$.

b.2. $V([\kappa]) \cap T_{2}=V([\kappa])$. In this case, $[\kappa] \notin\left[\mathbf{C}\left(\Gamma^{k}\right)\right]\left(T_{1} \cup T_{2}\right)$ and $[\kappa] \in\left[\mathbf{C}\left(\Gamma^{k}\right)\right]\left(T_{1} \cap T_{2}\right)$.

c. $[\kappa] \notin\left[\mathbf{C}\left(\Gamma^{k}\right)\right]\left(T_{1}\right)$ and $[\kappa] \in\left[\mathbf{C}\left(\Gamma^{k}\right)\right]\left(T_{2}\right)$. Analogously as in case (b), we distinguish between two new cases: $V([\kappa]) \cap T_{1}=\emptyset$ and $V([\kappa]) \cap T_{1}=V([\kappa])$.

c.1. $V([\kappa]) \cap T_{1}=\emptyset$. In this case, $[\kappa] \in\left[\mathbf{C}\left(\Gamma^{k}\right)\right]\left(T_{1} \cup T_{2}\right)$ and $[\kappa] \notin\left[\mathbf{C}\left(\Gamma^{k}\right)\right]\left(T_{1} \cap T_{2}\right)$.

c.2. $V([\kappa]) \cap T_{1}=V([\kappa])$. In this case, $[\kappa] \notin\left[\mathbf{C}\left(\Gamma^{k}\right)\right]\left(T_{1} \cup T_{2}\right)$ and $[\kappa] \in\left[\mathbf{C}\left(\Gamma^{k}\right)\right]\left(T_{1} \cap T_{2}\right)$.

d. $[\kappa] \notin\left[\mathbf{C}\left(\Gamma^{k}\right)\right]\left(T_{1}\right)$ and $[\kappa] \notin\left[\mathbf{C}\left(\Gamma^{k}\right)\right]\left(T_{2}\right)$. In this case, $[\kappa] \notin\left[\mathbf{C}\left(\Gamma^{k}\right)\right]\left(T_{1} \cup T_{2}\right)$ and $[\kappa] \notin$ $\left[\mathbf{C}\left(\Gamma^{k}\right)\right]\left(T_{1} \cap T_{2}\right)$. 
Following these cases, we have $\left|\left[\mathbf{C}\left(\Gamma^{k}\right)\right]\left(T_{1}\right)\right|+\left|\left[\mathbf{C}\left(\Gamma^{k}\right)\right]\left(T_{2}\right)\right|=\left|\left[\mathbf{C}\left(\Gamma^{k}\right)\right]\left(T_{1} \cup T_{2}\right)\right|+\left|\left[\mathbf{C}\left(\Gamma^{k}\right)\right]\left(T_{1} \cap T_{2}\right)\right|$ and, therefore,

$$
\begin{aligned}
\sum_{i \in T_{1}}\left|\left\{[\kappa] \in\left[\mathbf{C}\left(\Gamma^{k}\right)\right]: V([\kappa]) \cap T_{1}=\{i\}\right\}\right|+\sum_{i \in T_{2}}\left|\left\{[\kappa] \in\left[\mathbf{C}\left(\Gamma^{k}\right)\right]: V([\kappa]) \cap T_{2}=\{i\}\right\}\right| \\
=\left|\left\{[\kappa] \in\left[\mathbf{C}\left(\Gamma^{k}\right)\right]:\left|V([\kappa]) \cap T_{1}\right|=1\right\}\right|+\left|\left\{[\kappa] \in\left[\mathbf{C}\left(\Gamma^{k}\right)\right]:\left|V([\kappa]) \cap T_{2}\right|=1\right\}\right| \\
=\left|\left\{[\kappa] \in\left[\mathbf{C}\left(\Gamma^{k}\right)\right]:\left|V([\kappa]) \cap\left(T_{1} \cup T_{2}\right)\right|=1\right\}\right|+\left|\left\{[\kappa] \in\left[\mathbf{C}\left(\Gamma^{k}\right)\right]:\left|V([\kappa]) \cap\left(T_{1} \cap T_{2}\right)\right|=1\right\}\right| \\
=\sum_{i \in T_{1} \cup T_{2}}\left|\left\{[\kappa] \in\left[\mathbf{C}\left(\Gamma^{k}\right)\right]: V([\kappa]) \cap\left(T_{1} \cup T_{2}\right)=\{i\}\right\}\right| \\
\quad+\sum_{i \in T_{1} \cap T_{2}}\left|\left\{[\kappa] \in\left[\mathbf{C}\left(\Gamma^{k}\right)\right]: V([\kappa]) \cap\left(T_{1} \cap T_{2}\right)=\{i\}\right\}\right| .
\end{aligned}
$$

Proof of Lemma 4.5: By Lemma A.2 (ii), we have that $T_{1} \cup T_{2}, T_{1} \cap T_{2} \in \mathcal{E}\left(N_{0}, C\right)$. Then,

$$
\begin{aligned}
c^{*}\left(T_{1}\right)+c^{*}\left(T_{2}\right) & \\
= & k\left|T_{1}\right| \\
& -(K-k)\left(\sum_{i \in T_{1}}\left(\left|\left\{j \in N_{0} \backslash T_{1}:\{i, j\} \in F^{k}\right\}\right|+\left|\left\{[\kappa] \in\left[\mathbf{C}\left(\Gamma^{k}\right)\right]: V([\kappa]) \cap T_{1}=\{i\}\right\}\right|\right)-1\right) \\
& +k\left|T_{2}\right| \\
& -(K-k)\left(\sum_{i \in T_{2}}\left(\left|\left\{j \in N_{0} \backslash T_{2}:\{i, j\} \in F^{k}\right\}\right|+\left|\left\{[\kappa] \in\left[\mathbf{C}\left(\Gamma^{k}\right)\right]: V([\kappa]) \cap T_{2}=\{i\}\right\}\right|\right)-1\right) \\
= & k\left(\left|T_{1}\right|+\left|T_{2}\right|\right) \\
& -(K-k)\left(\sum_{i \in T_{1}}\left(\left|\left\{j \in N_{0} \backslash T_{1}:\{i, j\} \in F^{k}\right\}\right|+\left|\left\{[\kappa] \in\left[\mathbf{C}\left(\Gamma^{k}\right)\right]: V([\kappa]) \cap T_{1}=\{i\}\right\}\right|\right)-1\right) \\
& -(K-k)\left(\sum_{i \in T_{2}}\left(\left|\left\{j \in N_{0} \backslash T_{2}:\{i, j\} \in F^{k}\right\}\right|+\left|\left\{[\kappa] \in\left[\mathbf{C}\left(\Gamma^{k}\right)\right]: V([\kappa]) \cap T_{2}=\{i\}\right\}\right|\right)-1\right) \\
= & k\left|T_{1} \cup T_{2}\right|-(K-k)\left(\sum _ { i \in T _ { 1 } \cup T _ { 2 } } \left(\left|\left\{j \in N_{0} \backslash\left(T_{1} \cup T_{2}\right):\{i, j\} \in F^{k}\right\}\right|\right.\right. \\
& \left.\left.+\left|\left\{[\kappa] \in\left[\mathbf{C}\left(\Gamma^{k}\right)\right]: V([\kappa]) \cap\left(T_{1} \cup T_{2}\right)=\{i\}\right\}\right|\right)-1\right) \\
& +k\left|T_{1} \cap T_{2}\right|-(K-k)\left(\sum _ { i \in T _ { 1 } \cap T _ { 2 } } \left(\left|\left\{j \in N_{0} \backslash\left(T_{1} \cap T_{2}\right):\{i, j\} \in F^{k}\right\}\right|\right.\right. \\
& \left.\left.+\left|\left\{[\kappa] \in\left[\mathbf{C}\left(\Gamma^{k}\right)\right]: V([\kappa]) \cap\left(T_{1} \cap T_{2}\right)=\{i\}\right\}\right|\right)-1\right) \\
& =c^{*}\left(T_{1} \cup T_{2}\right)+c^{*}\left(T_{1} \cap T_{2}\right), \quad
\end{aligned}
$$


where the first and last equalities follow from Lemma A.1 and the third equality is a direct consequence of Lemma A.3 (ii) and (iii).

\section{References}

Brânzei, R., Iñarra, E., Tijs, S. and Zarzuelo, J. (2006), 'A simple algorithm for the nucleolus of airport profit problems', International Journal of Game Theory 34, 259-272.

Claus, A. and Kleitman, D. J. (1973), 'Cost-allocation for a spanning tree', Networks 3, $289-304$.

Derks, J. and Kuipers, J. (1997), 'On the core of routing games', International Journal of Game Theory 26, 193-205.

Estévez-Fernández, A. (2012), 'New characterizations for largeness of the core', Games and Economic Behavior 76, 160-180.

Estévez-Fernández, A., Borm, P., Meertens, M. and Reijnierse, H. (2009), 'On the core of routing games with revenues', International Journal of Game Theory 38, 291-304.

Gillies, D. (1953), Some theorems on n-person games, PhD thesis, Princeton, University Press Princeton, New Jersey.

Granot, D. and Huberman, G. (1981), 'Minimum cost spanning tree games', Mathematical Programming 21, 1-18.

Granot, D. and Huberman, G. (1984), 'On the core and nucleolus of minimum cost spanning tree games', Mathematical Programming 29, 323-347.

Kuipers, J. (1994), Combinatorial methods in cooperative game theory, PhD thesis, Maastricht University. I.S.B.N. 90-5278-141-9.

Kuipers, J., Solymosi, T. and Aarts, H. (2000), 'Computing the nucleolus of some combinatorially structured games', Mathematical Programming 88, 541-563.

Littlechild, S. C. and Owen, G. (1976), 'A further note on the nucleolus of the "airport game", International Journal of Game Theory 5, 91-95.

Meertens, M. and Potters, J. (2006), 'The nucleolus of trees with revenues', Mathematical Methods of Operations Research 64, 363-382. 
Moretti, S. (2008), Cost allocation problems arising from connection situations in an interactive cooperative setting, PhD thesis, Tilburg University. I.S.B.N. 978-90-5668-218-7.

Potters, J. A., Curiel, I. J. and Tijs, S. H. (1992), 'Traveling salesman games', Mathematical Programming 53, 199-211.

Schmeidler, D. (1972), 'Cores of exact games', Journal of Mathematical Analysis and Applications 40, 214225.

Sharkey, W. (1982), 'Cooperative games with large cores', Int. J. Game Theory 11, 175-182.

Suijs, J., Borm, P., Hamers, H., Quant, M. and Koster, M. (2005), 'Communication and cooperation in public network situations', Annals of Operations Research 137, 117-140. 